Article

\title{
Analysis of Ripple Current in the Capacitors of Active Power Filters
}

\author{
Tadeusz Platek \\ Institute of Control and Industrial Electronic, Warsaw University of Technology, 75 Koszykowa St., \\ 00-662 Warsaw, Poland; tadekplatek@o2.pl; Tel.: +48-223-144-200
}

Received: 18 October 2019; Accepted: 18 November 2019; Published: 25 November 2019

check for updates

\begin{abstract}
This article provides formulae to determine the root mean square (rms) value of a capacitor current in an inductive-capacitive-inductive (LCL) filter used in a parallel active power filter (PAPF) circuit. The article presents an analysis of three components of the capacitor current: a component forced by the usually distorted voltage of the grid; a component forced by the nonlinear load current harmonics and harmonics in the output current of the PAPF that compensates them (a novel aspect presented in this document); and a component forced by the inverters of the PAPF containing carrier and sideband harmonics. The article also presents formulae for determining the rms value of current harmonics in dc-link capacitors forced by the ripple of the ac output current without load of the filter inverters (also novel to this document). The results of the analysis have been confirmed by simulation and experimental research of a commercial active filter consisting of two parallel interleaved voltage inverters. Elements of the LCL filter of the PAPF have been selected according to dependencies available in scientific and technical literature. In addition, the formulae presented in the article are used to verify the correctness of selection of capacitors from the point of view of their catalogue acceptable rms value of capacitor current.
\end{abstract}

Keywords: parallel active power filter; bessel functions; asymmetrical regular sampled PWM; LCL-ripple filter; dc-link capacitor

\section{Introduction}

The parallel active power filter (PAPF) contains three groups of passive power elements: dc-link capacitors, inductances, and capacitors in the LCL-ripple filter. Specified in the data sheet, the permissible capacitor rms current is one of its basic parameters. Liserre et al. [1] propose a step-by-step procedure for designing the LCL filter of a front-end three-phase active rectifier. Jeong et al. in [2] and Asiminoei et al. in [3] give formulae allowing for calculation of rms current ripple in the LCL inductors. In [4], Jalili and Bernet applied Bessel functions to select the LCL filter parameters for defined maximum grid current harmonics.

This article presents an analysis leading to the determination of the rms current of LCL-ripple filter capacitors used at the output of the PAPF. There are three components of this current:

(1) Component forced by the usually distorted mains voltage;

(2) Component forced by the nonlinear load current harmonics and harmonics in the output current of the PAPF that compensates them;

(3) Component forced by the inverters of the PAPF and containing carrier and sideband harmonics (for equal and different inductances on the converter side).

In systems with a single inverter, the dominant harmonic frequency of the output voltage is equal to the frequency of the auxiliary carrier harmonic. Sahoo et al. [5] give the relation determining 
the rms current of the LCL filter capacitor in a one-grid connected inverter for the first component (for an undistorted grid) and the third component of this current. In PAPF systems with two parallel interleaved inverters, "carrier harmonics" disappear; thus, the share of the second component in the rms current of the capacitor increases.

Studies describing the rms current of the LCL-capacitor ripple of output filter installed at the output of parallel interleaved inverters are not common in the scientific literature. Tang et al. in [6] and Vodyakho et al. in [7] analyze the impact of capacitance values of LCL filters on the stability of the PAPF.

This article presents the equations describing the rms value of the second component, independent of the number of parallel interleaved inverters, and the rms value of the third component for two parallel interleaved inverters. Formulae are given for the PAPF containing a transformer with a Dy5 connection group.

The ripple currents in the LCL capacitors, LCL inductors, and dc-link capacitors depend on each other [3]. This article provides formulae to determine the rms value of the ripple current in dc-link capacitors, which comes from the ripple current in the LCL inductors. McGrath et al. in [8], Kolar et al. in [9], Zhang et al. in $[10,11]$, provide an algorithm for calculating the value of the harmonic current in the dc-link capacitor for the voltage source when the load current is assumed to be a fundamental single-frequency sine. The analysis presented in these works, applied to the PAPF system with the deactivated reactive power compensation for the basic harmonic, will not reveal the harmonic values determined in this article, even if it takes into account the 5-, 7-, 11-, and 13-order ac harmonic in the output current. Zhang et al. in [11], Ye et al. in [12], and Quan at al. [13] proposed an interleaving scheme to reduce dc-link current harmonics, but the optimization of the interleaving angle from the point of view of harmonic currents in the dc-circuit also influences the ripple current in the ac capacitors.

Many articles publish theoretical analysis and results of simulation and experimental tests of current components in LCL filter capacitors and dc-link circuit. However, they do not provide dependencies which can be easily used to easily calculate the rms values of currents in these capacitors. This article gives simplified equations with an error of about $10 \%$ on the basis of which one can determine both the rms value of the capacitor current in the dc-link circuit and the three current components of the LCL filter capacitor.

In the present paper, the rms values of currents in LCL filter capacitors and dc-link capacitors was determined analytically for the control system of the filter output current with asymmetrical regular-sampled PWM. In addition, simulation tests for control with symmetrical regular-sampled PWM have been conducted.

\section{Description of the Input Circuit of PAPF}

Figure 1a,b shows the schematic diagrams of the analyzed medium-voltage power supply system. A controlled twelve-pulse converter is powered via a transformer Tr 2 with a vector group of Dd0y5 connections. A PAPF containing two voltage inverters and a medium voltage transformer $\operatorname{Tr} 1$ with a vector group of Dy 5 compensates harmonic current drawn by the non-linear load in the form of a 12Th converter (Th1-Th12). Two capacitors with capacitance $C / 2$ at the output of each phase function as a capacitor with capacitance $C$ of the LCL filter reducing common mode voltage (CMV) [14]. This connection of capacitors ensures the free flow of harmonics of their zero sequence currents to the $d c$-circuit. The chokes $L_{-} 1, L_{-2}$ are installed at the output of the inverters and, together with the capacitors with the resultant capacitance $C$ and the leakage inductance of the transformer Tr1, create an LCL type filter $[6,15-19]$ in each phase. $R_{d}$ is LCL-filter resistor damping. 


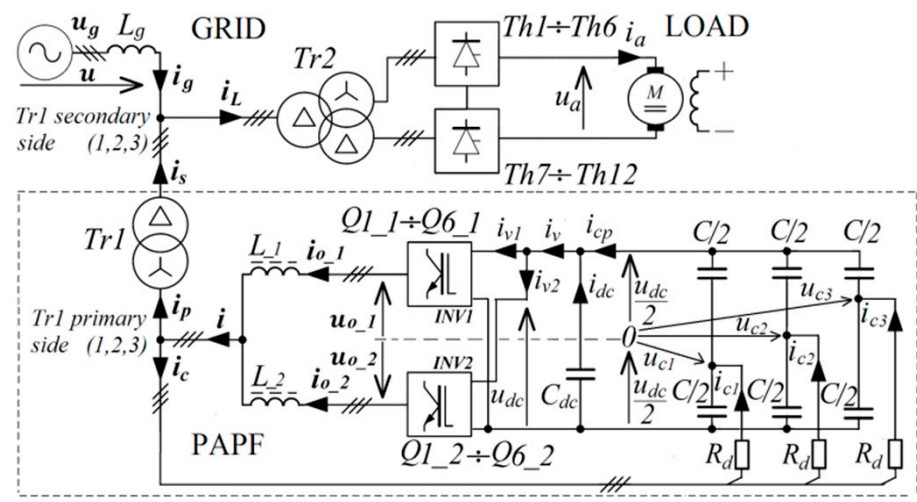

(a)

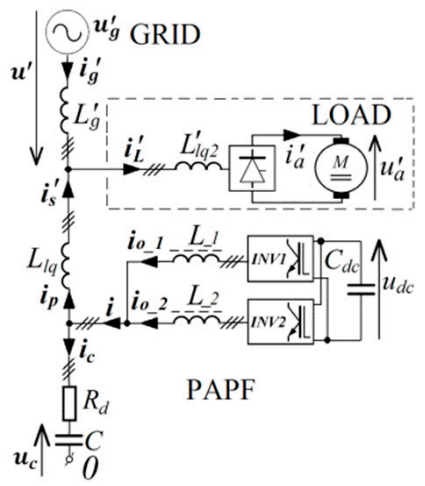

(b)

Figure 1. (a) Diagram of circuit with PAPF and (b) its simplified version.

The PAPF has two inverters $\left(Q_{1 \_1}-Q_{6 \_}\right)$[3] with FF600R12IS4F hybrid modules (combination of Insulated Gate Bipolar Transistor (IGBT) with silicon carbide Schottky diodes).

Voltages and currents $\boldsymbol{u}_{g}, \boldsymbol{u}, \boldsymbol{i}_{g}, \boldsymbol{i}_{L}, \boldsymbol{i}_{s}, \boldsymbol{i}_{p}, \boldsymbol{i}_{c}, \boldsymbol{i}_{\mathrm{O} \_1}, \boldsymbol{i}_{0_{-} 2}$, and $\boldsymbol{i}$ shown in Figure $1 \mathrm{a}, \mathrm{b}$ are vectors: $\boldsymbol{u}_{\boldsymbol{g}}=\left[u_{g 1}, u_{g 2}, u_{g 3}\right]^{T} ; \boldsymbol{u}=\left[u_{1}, u_{2}, u_{3}\right]^{T} ; \boldsymbol{u}_{c}=\left[u_{c 1}, u_{c 2}, u_{c 3}\right]^{T} ; \boldsymbol{i}_{\boldsymbol{g}}=\left[i_{g 1}, i_{g 2}, i_{g 3}\right]^{T} ; \boldsymbol{i}_{\boldsymbol{L}}=\left[i_{L 1}, i_{L 2,}, i_{L 3}\right]^{T} ; \boldsymbol{i}_{s}=\left[i_{s 1}, i_{s 2}, i_{s 3}\right]^{T} ;$

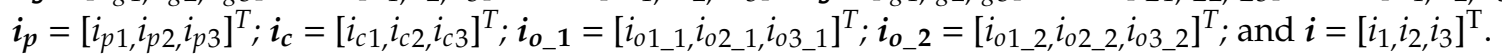

Table 1 shows the relation of the primary and secondary vectors of the Dy 5 transformer voltage (where $v_{z}$ is the transformer turns ratio). Vectors $u^{\prime}{ }_{g}, u^{\prime}, i^{\prime}{ }_{g^{\prime}}, i_{L}^{\prime}$, and $i_{s}^{\prime}$ are values brought to transformer $\operatorname{Tr} 1$ primary side terminals $1,2,3$. Reference [19] gives the relations between the primary and the secondary vectors of the Dy11 transformer voltage.

Table 1. Relation of primary and secondary vectors of Dy5 transformer voltage.

\begin{tabular}{lccccc}
\hline Relation for $u_{g}^{\prime}$ & Relation for $u^{\prime}$ & Relation for $i_{g}^{\prime}$ & Relation for $i_{L}^{\prime}$ & Relation for $i_{s}^{\prime}$ & Matrix A \\
\hline$u_{g}^{\prime}=-\frac{1}{v_{z}} A u_{g}$ & $u^{\prime}=-\frac{1}{v_{z}} A u$ & $i_{g}^{\prime}=\frac{v_{z}}{3} A i_{g}$ & $i_{L}^{\prime}=-\frac{v_{z}}{3} A i_{L}$ & $i_{s}^{\prime}=-\frac{v_{z}}{3} A i_{s}$ & $\mathbf{A}=\left[\begin{array}{ccc}1 & -1 & 0 \\
0 & 1 & -1 \\
-1 & 0 & 1\end{array}\right]$ \\
\hline
\end{tabular}

The algorithm described in the literature $[20,21]$ is implemented in the control system with a voltage regulator in the $d c$-circuit. The control block diagram, criteria for selection of the current regulator gain factor, and criteria for determining the inductance $L$ at the outputs of the PAPF ( $L$ is the nominal value of $L_{-1}$ and $L_{-2}$ ) are presented in [22]. The analysis carried out in this article does not influence the impact of PAPF control methods on the quality of compensation of higher harmonics of non-linear load current.

Commonly applied in power electronics, a TMS320F28335 Digital Signal Processor was used to implement the chosen filter control.

\section{Inverter Output Voltage with Asymmetrical Regular-Sampled PWM}

Equations describing the output voltage of the transistor branch $u_{o i_{j} j}$ produced in the system with asymmetrical regular-sampled PWM are given in [23]:

$$
u_{o i_{-} j}=\sum_{n=1}^{\infty} F_{0 n} \cos \left(n \omega_{g} t+n \theta_{g i}^{\prime}\right)+\sum_{m=1}^{\infty} \sum_{n=-\infty}^{\infty} F_{m n} \cos \left[\left(m \omega_{C} / \omega_{g}+n\right) \omega_{g} t+m \theta_{C i_{-} j}+n \theta_{g i}^{\prime}\right]
$$

where indexes $i \in\{1,2,3\}, j \in\{1,2\}$ denote the inverter's branch and number, respectively; $\theta_{g i^{\prime}}^{\prime} \theta_{C i_{-} j}$ are the phase angles of the fundamental harmonic of the mains voltage $u_{g}$ brought to transformer $\operatorname{Tr} 1$ primary side terminals $1,2,3$, and a carrier wave, respectively; $\omega_{C}$ is the pulsation of the triangular 
carrier; $\omega_{g}$ is the angular frequency of the fundamental harmonic of line voltage $u_{g}$; and $m$ is the carrier index variable. Harmonic coefficients $F_{m n}$ are given in [23] by:

$$
F_{m n}=\frac{2 U_{d c}}{\pi} \frac{J_{n}\left(\left[m+n \frac{\omega_{g}}{\omega_{C}}\right] \frac{\pi M}{2}\right)}{m+n \frac{\omega_{g}}{\omega_{C}}} \sin \left[(m+n) \frac{\pi}{2}\right]
$$

where $J_{n}(\mathrm{x})$ denotes a Bessel function of the first type, with order $n$ and argument $x ; U_{d c}$ is dc-link voltage; $M$ is modulation depth $\left(M \approx 2 \hat{U}_{g, 1}^{\prime} / U_{d c}, 0 \leq M \leq 1\right)$; and $\hat{U}_{g, 1}^{\prime}$ is the amplitude of the fundamental harmonic voltages $u_{g 1,1}^{\prime}, u_{g 2,1}^{\prime}$ and $u_{g 3,1}^{\prime}$. The form of Equation (2) indicates that $F_{m n}$ coefficient takes a zero value if the conditions of $m+n=0, \pm 2, \pm 4 \ldots$ are fulfilled.

From Equations (1) and (2) it can be concluded that the carrier and sideband harmonics of the same order set for $\theta_{C i_{-j}}=0$ or $\theta_{C i_{-j}}=\pi$ have the same amplitudes and their phase values are equal or differ by $\pi \mathrm{rad}$. If the active filter includes two inverters connected as shown in Figure 1a, from the point of view of the higher harmonics in the output current of the filter it is advantageous to offset the carriers from each other in modulator systems that control the transistor in the respective phase legs of the inverters by an angle $\pi$ rad [3,24]. After insertion of $\theta_{C_{-} i_{1}}$ into Equation (1) for $j=1$ and then substituting $\theta_{C i \_2}=\theta_{C_{-} 1}+\pi$ in Equation (1) for $j=2$, we can obtain the following:

$$
\begin{aligned}
& u_{o i \_1}=\sum_{n=1}^{\infty} U_{o_{-} 1,0 n} \cos \left(n \omega_{g} t+n \theta^{\prime}{ }_{g i}\right)+\sum_{m=1}^{\infty} \sum_{n=-\infty}^{\infty} U_{o_{-} 1, m n} \cos \left\{\left[m\left(\omega_{C} / \omega_{g}\right)+n\right] \omega_{g} t+m \theta_{C i_{-} 1}+n \theta^{\prime}{ }_{g i}\right\} \\
& u_{o i \_}=\sum_{n=1}^{\infty} U_{o_{-} 2,0 n} \cos \left(n \omega_{g} t+n \theta^{\prime}{ }_{g i}\right)+\sum_{m=1}^{\infty} \sum_{n=-\infty}^{\infty} U_{o_{-} 2, m n} \cos \left\{\left[m\left(\omega_{C} / \omega_{g}\right)+n\right] \omega_{g} t+m \theta_{C i_{-} 1}+n \theta^{\prime}{ }_{g i}\right\}
\end{aligned}
$$

Harmonic coefficients $U_{o_{-} 1, m n}$ and $U_{o_{-}, m n}$ are given by

$$
\begin{gathered}
U_{o_{-} 1, m n}=F_{m n} \\
U_{o_{-} 2, m n}=F_{m n}(-1)^{m}
\end{gathered}
$$

If we assume that $\omega_{C} / \omega_{g} \geq 40$, then the coefficients of subharmonics $U_{o_{-} 1, m n}$ or $U_{0_{-} 2, m n}$ present for non-integer values of quotient $\omega_{C} / \omega_{g}$ values will be very small and their impact on the effective value of the capacitor current will be negligible. Therefore, further considerations can be carried out assuming that the waveforms $u_{o i_{-} 1}$ and $u_{o i_{-} 2}$ are the periodic functions, which means that the size of $\xi$, defined by formula $\xi=\omega_{C} / \omega_{g}$ is an integer. Harmonic coefficients $U_{o_{-} 1, m n}$ denoted by $U_{o_{-} 1, k}, U_{o_{-} 2, m n}$ will also be denoted by $U_{o_{-} 2, k}$ where $k=m \xi+n$. The choice of $\omega_{C} / \omega_{g} \geq 40$ is justified by the limit values of inequalities (17) given in Section 4.3. Figure 2 shows the harmonics spectrum of the output voltages expressed in the p.u. system of each inverter with asymmetrical regular-sampled PWM. Amplitudes of voltage harmonic coefficients have been determined in relation to half the voltage of the $d c$-link circuit $\left(\hat{U}_{o, k(p u)}=2 \hat{U}_{o, k} / U_{d c}\right)$.

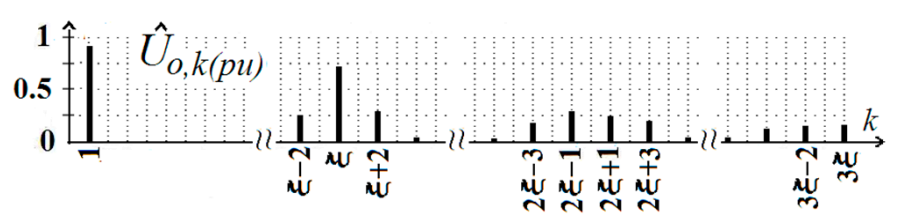

Figure 2. Harmonics spectrum of output voltages of each branch of inverters $(M=0.9)$.

The spectrum of $u_{o i_{-}}$shows that significant harmonics of the output currents of the branches of inverters occur for $m=1$ if $n= \pm 2$, and for $m=2$ if $n= \pm 1, \pm 3(|n| \leq 3)$. 


\section{LCL System Capacitor Current}

The analysis in this article will be carried out assuming full symmetry of both the load and the three-phase supply voltage.

For clarity of the following considerations, the vector magnitudes were replaced by their components without indices " 1 ", " 2 ", and " 3 ".

In the power supply system with the PAPF containing a transformer, determining the rms values of the LCL-ripple filter capacitor current requires calculation of amplitudes and initial phases of mains voltage harmonics $u_{g, k}$ and of load current $i_{L, k}$ brought to transformer $\operatorname{Tr} 1$ primary side terminals 1, 2, and 3.

The following relations can describe the $k$-th order harmonic of voltage $u_{g, k}$, and current $i_{L, k}$ :

$$
\begin{gathered}
u_{g, k}=\hat{U}_{g, k} \sin \left(k \omega_{g} t+\psi_{g, k}\right) \\
i_{L, k}=\hat{I}_{L, k} \sin \left(k \omega_{g} t+\varphi_{L, k}\right)
\end{gathered}
$$

where $\psi_{g, k}, \varphi_{L, k}, \hat{U}_{g, k}, \hat{I}_{L, k}$ are the initial phases and amplitudes of the $k$-th harmonic voltage $u_{g, k}$ and current $i_{L, k}$, respectively.

The formulae presented in Table 1 allow determination of the amplitudes $\left(\hat{U}_{g, k^{\prime}}^{\prime} \hat{I}_{L, k}^{\prime}\right)$ and the initial phases $\left(\psi_{g, k^{\prime}}^{\prime} \varphi_{L, k}^{\prime}\right)$ of the harmonic voltage $u_{g, k}^{\prime}$ and current $i_{L, k^{\prime}}^{\prime}$ respectively, for the system with a transformer of a group of Dy 5 connections. These relationships are given in Table 2.

Table 2. The relation of the primary and the secondary values of Dy5 transformer voltage.

\begin{tabular}{cccc}
\hline Relation for $\hat{U}_{g, k}^{\prime}$ & Relation for $\psi_{g, k}^{\prime}$ & Relation for $\hat{I}_{L, k}^{\prime}$ & Relation for $\varphi_{L, k}^{\prime}$ \\
\hline$\hat{U}_{g, k}^{\prime}=\frac{\sqrt{3} \hat{U}_{g, k}}{v_{z}}$ & $\psi_{g, k}^{\prime}=\psi_{g, k}+\frac{5 \pi}{6}$ & $\hat{I}_{L, k}^{\prime}=v_{z} \frac{\hat{L}_{L, k}}{\sqrt{3}}$ & $\varphi_{L, k}^{\prime}=\varphi_{L, k}+\frac{5 \pi}{6}$ \\
\hline
\end{tabular}

We can select three components of the LCL filter capacitor current based on the sources that force them:

$$
i_{c}=i_{c G}+i_{c L}+i_{c C}
$$

where the $i_{c G}$ component is forced by the usually distorted voltage of mains supply; the $i_{c L}$ component is forced by the current harmonics of nonlinear load and the harmonics in the output current of the PAPF that compensates them and the component $i_{c C}$ is also forced by the PAPF and contains the carrier (for $L_{-} 1 \neq L_{-}$) and sideband harmonics.

In real conditions, the number of measured (by typical Total Harmonic Distortion measurement) harmonics does not exceed 40. For the determination of the first two components of the rms current of the capacitor, it was assumed that the voltage inverters with inductances $L_{-} 1$ and $L_{-2}$ at the output can be replaced with current sources for harmonics of an order below 40 .

\subsection{Rms of Capacitor C Current Component $I_{c G, r m s}$}

The frequency spectrum of the capacitor current component $i_{c G}$ contains fundamental and baseband harmonics $k=n=1,2 \ldots(m=0)$.

The first of these components can be determined from the equivalent circuit shown in Figure 3a. Based on this diagram, we can determine the amplitude $\hat{I}_{c G, k}$ :

$$
\hat{I}_{c G, k}=\frac{\hat{U}_{g, k}}{\sqrt{\left[k \omega_{g}\left(L_{l q}+L_{g}^{\prime}\right)-1 /\left(k \omega_{g} C\right)\right]^{2}+R_{d}^{2}}}
$$


where $L_{l q}$ is the dispersion inductance $\operatorname{Tr} 1$, and $L_{g}^{\prime}$ is the grid-side inductance related to the low voltage side of $\operatorname{Tr} 1$. The damping resistor $R_{d}$ was determined on the basis of the relation given in [25]:

$$
R_{d}=2 \zeta_{p} \sqrt{\frac{\left(L_{l q}+L_{g}^{\prime}\right) L}{\left[2\left(L_{l q}+L_{g}^{\prime}\right)+L\right] C}}
$$

where $\zeta_{p}$ is the resonant-pole damping factor, and $L$ is the nominal value of $L_{-1}$ and $L_{-2}\left(L_{-1} \approx L_{-}\right)$.

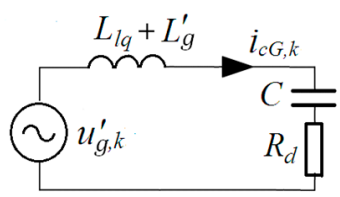

(a)

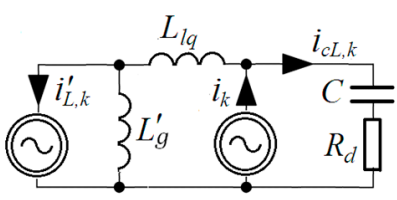

(b)

Figure 3. Equivalent diagram of LCL circuit in a filter system with two inverters: (a) for the current component forced by the $a c$ mains and (b) for the current component forced by load.

The damping factor has been considered critical in some theoretical works [26].

\subsection{Rms of Capacitor C Current Component $I_{c L, r m s}$}

The frequency spectrum of the capacitor current component $i_{c L}$ contains fundamental and baseband harmonics of the order $k=n=1,2 \ldots(m=0)$.

The amplitudes of particular harmonics of the second component can be determined on the basis of the scheme shown in Figure 3b:

$$
\hat{I}_{c L, k}=\left|\frac{k^{2} \omega_{g}^{2} C\left[\hat{I}_{k}\left(L_{g}^{\prime}+L_{l q}\right)-\hat{I}_{L, k}^{\prime} L_{g}^{\prime}\right]}{\sqrt{\left[1-k^{2} \omega_{g}^{2} C\left(L_{l q}+L_{g}^{\prime}\right)\right]^{2}+\left(k \omega_{g} C R_{d}\right)^{2}}}\right|
$$

where $\hat{I}_{L, k}^{\prime}$ and $\hat{I}_{k}$ are the amplitudes of the $k$-order harmonic of the load current and the component of the filter output current compensating this harmonic, respectively. If the harmonic attenuation factor $\lambda$ of the $k$-th harmonic of load current $\left(\lambda_{k}=\hat{I}_{k} / \hat{I}_{L, k}^{\prime}\right)$ is known, we obtain:

$$
\hat{I}_{c L, k}=\left|\frac{k^{2} \omega_{g}^{2} C\left[\lambda_{k}\left(L_{g}^{\prime}+L_{l q}\right)-L_{g}^{\prime}\right]}{\sqrt{\left[1-k^{2} \omega_{g}^{2} C\left(L_{l q}+L_{g}^{\prime}\right)\right]^{2}+\left(k \omega_{g} C R_{d}\right)^{2}}}\right| \hat{I}_{L, k}^{\prime}
$$

$\lambda_{k}$ is usually a set point parameter in the PAPF.

Harmonic components of $i_{c G}$ and $i_{c L}$ may be of the same order. The resulting harmonic of this order depends on the amplitude and initial phase of harmonic components. In practical systems, the measurement of the amplitude and the initial phase of mains voltage harmonics should be made with the load and PAPF switched off.

Current $i_{c G, k}$ forced by the $k$-th harmonic voltage $u_{g, k}$ is capacitive if $k$ satisfies the following inequality:

$$
k<1 /\left(\omega_{g} \sqrt{\left(L_{l q}+L_{g}^{\prime}\right) C}\right)
$$

The rms value of $I_{c G L, r m s}$, considering the $i_{c G}$ and $i_{c L}$ components, is described by the formula

$$
I_{c G L, r m s}=\sqrt{\sum_{k=1}^{\infty}\left[0.5\left(\hat{I}_{c G, k}^{2}+\hat{I}_{c L, k}^{2}\right) \pm \operatorname{sign}\left[\lambda_{k}\left(L_{g}^{\prime}+L_{l q}\right)-L_{g}^{\prime}\right] \hat{I}_{c G, k} \hat{I}_{c L, k} \sin \left(\Delta \varphi_{k}\right)\right]}
$$


where $\Delta \varphi_{k}=\psi_{g, k}-\varphi_{L, k}=\psi_{g, k}^{\prime}-\varphi_{L, k}^{\prime}$. In Equation (13) "+" symbolizes the inductive and "-" the capacitive nature of the current $i_{c G, k}$ (respectively). If the initial phases $\psi_{g, k}$ and $\varphi_{L, k}$ are not known the equation used is

$$
I_{c G L, r m s} \leq \sqrt{0.5 \sum_{k=1}^{\infty}\left(\hat{I}_{c G, k}+\hat{I}_{c L, k}\right)^{2}}
$$

\subsection{Rms of Capacitor C Current Ripple $I_{c C, r m s}$}

The frequency spectrum of the capacitor ripple current contains carrier harmonics of the $k=\xi+n$ order (for $L_{-} 1 \neq L_{-2}$ ) and sideband harmonics of the $k=m \xi+n(m=2,4,6 \ldots)$ order.

Figure $4 \mathrm{a}, \mathrm{b}$ show carrier waveforms for two PWM techniques: (Figure $4 \mathrm{a}$ ) double interleaved PWM carriers [3] and (Figure 4b) double three interleaved PWM carriers [13,22].

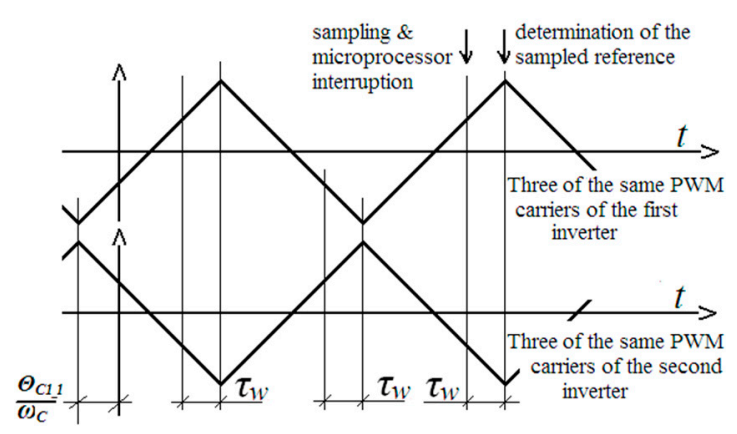

(a)

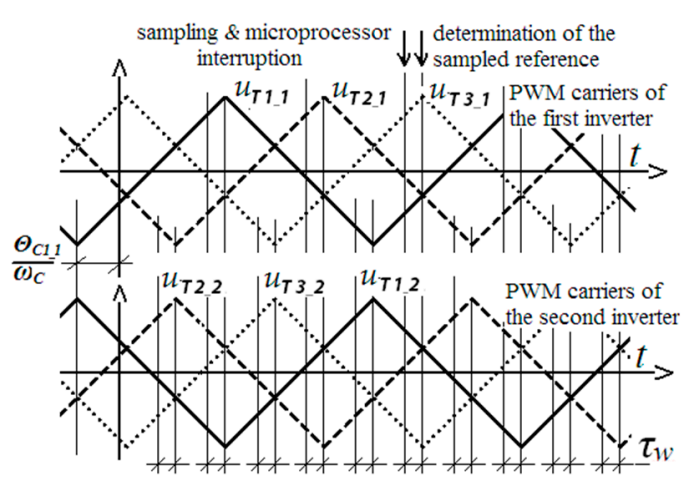

(b)

Figure 4. Carrier waveforms for double PWM techniques. (a) Double Interleaved PWM Carriers [3] and (b) Double Three Interleaved PWM Carriers [13,22].

The duration time of the microprocessor interruption service routine is denoted by $\tau_{w}$ (PWM computation delays [22,27]). The choice of the PWM technique is justified by the results of the simulation research collected in Section 6.3. These studies show that double three interleaved PWM carriers ensure minimum values of common mode voltage (CMV), minimum values of the number of operations in the microprocessor interruption service routine $(\mathrm{N})$, and minimum values of rms current dc-circuit capacitors in the near-full range of the depth modulation index $\mathrm{M}$.

After insertion of $\theta_{C 1-1}$ in Equation (1) and then substituting phases angle $\theta_{\mathrm{C} 2-1}=\theta_{C_{1-1}}-2 \pi / 3$, $\theta_{C 3-1}=\theta_{C 1-1}+2 \pi / 3, \theta_{C i-2}=\theta_{C i-1}+\pi($ for $i=1,2,3), \theta_{g 1}^{\prime}=0, \theta_{g 2}^{\prime}=-2 \pi / 3, \theta_{g 3}^{\prime}=2 \pi / 3$, respectively, we obtain six equations, from which the following conclusion can be drawn: If the fundamental harmonics $u_{g 1,1}^{\prime}, u_{g 2,1}^{\prime}, u_{g 3,1}^{\prime}$ of voltages and carrier signals of the first, second and third branch of each inverter create the same, three-phase positive sequences, then the individual harmonics of the output voltages of each inverter $\left(u_{01-1}, u_{02}-1, u_{03}-1\right.$ and $\left.u_{01-2}, u_{02} 2, u_{03 \_}\right)$make a positive, negative or zero sequence if the conditions in Table 3 are met. The same rules also apply if the fundamental harmonics $u_{g 1,1}^{\prime}, u_{g 2,1}^{\prime}, u_{g 3,1}^{\prime}$ of voltages and carrier signals simultaneously form the same, three-phase negative sequences.

Table 3. Conditions for the type of three-phase harmonic sequence of inverter output voltages.

\begin{tabular}{|c|c|c|c|}
\hline$\left(u_{g 1,1}^{\prime}, u_{g 2,1}^{\prime}, u_{g 3,1}^{\prime}\right)$ and $\left(u_{T} 1_{-}, 2_{-}, 3_{-}\right)$ & \multicolumn{3}{|c|}{ 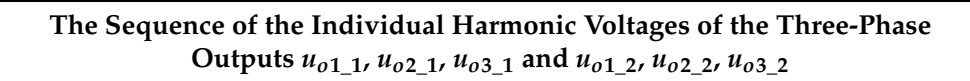 } \\
\hline- & positive sequence $(\mathrm{p})$ & negative sequence $(\mathrm{n})$ & zero sequence $(0)$ \\
\hline the same sequence & $m+n=-5,-2,1,4,7 \ldots$ & $m+n=-4,-1,2,5 \ldots$ & $m+n=-6,-3,0,3,6 \ldots$ \\
\hline opposite sequence & $m-n=-4,-1,2,5$. & $m-n=-5,-2,1,4,7$. & $m-n=-6,-3,0,3,6 \ldots$ \\
\hline
\end{tabular}


If we assume $\theta_{C 2-1}=\theta_{C 1-1}+2 \pi / 3, \theta_{C 3-1}=\theta_{C 1-1}-2 \pi / 3, \theta_{C i-2}=\theta_{C i-1}+\pi($ for $i=1,2,3), \theta_{g 1}^{\prime}$ $=0, \theta_{g 2}^{\prime}=-2 \pi / 3, \theta_{g 3}^{\prime}=2 \pi / 3$ then the fundamental harmonics $u_{g 1,1}^{\prime}, u_{g 2,1}^{\prime}, u_{g 3,1}^{\prime}$ of voltages form a three-phase positive sequence and carrier signals form a three-phase negative sequence. In the case of the opposite three-phase sequences $u_{g 1,1}^{\prime}, u_{g 2,1}^{\prime}, u_{g 3,1}^{\prime}$ of voltages to the sequence of carrier signals, the other rules are presented in Table 3. In this article, the relations for which the sequence of fundamental harmonics $u_{g 1,1}^{\prime}, u_{g 2,1}^{\prime}, u_{g 3,1}^{\prime}$ of voltages opposite to the sequence of carrier signals will be marked by superscript (op).

The same rules apply to the harmonic of capacitor currents $i_{c 1}, i_{c 2}$, and $i_{c 3}$ and output currents of branches of each inverter $i_{01} \_1, i_{02} 1, i_{03} 1, i_{01} 2, i_{02} 2$, and $i_{03} 2$, because the harmonic of the output voltages of each inverter $u_{01 \_}, u_{02 \_1}, u_{03 \_1}$ and $u_{01 \_2}, u_{02} 2$, and $u_{03 \_2}$ directly enforce the harmonics of these currents.

Figure 5a shows the equivalent circuit of the output circuit of the respective branches of the two inverters, based on which the $\hat{I}_{c C, k}$ amplitude of the $k$-th harmonic of the $C$ capacitor current can be determined if the harmonics of the currents $i_{c C 1, k}, i_{c C 2, k}$ and $i_{c C 3, k}$ form three-phase positive or negative sequences. As harmonics with three-phase zero sequence of $i_{01, k}$ and $i_{02, k}$ currents cannot flow in windings without the neutral wire of the transformer $\operatorname{Tr} 1$, the equivalent circuit shown in Figure 5a can be simplified for harmonics with zero sequence as shown in Figure 5b.

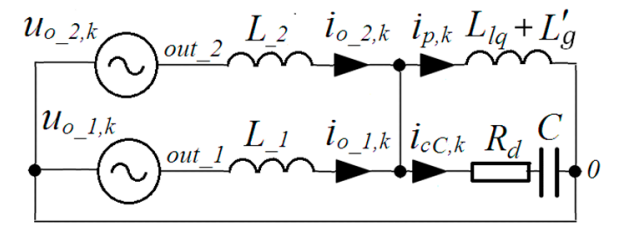

(a)

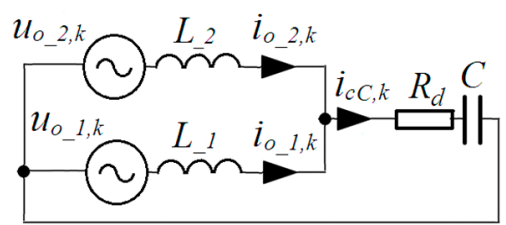

(b)

Figure 5. (a) Equivalent diagram of LCL circuit in an PAPF with two inverters for the current component $i_{c C, k}$ for the described control for positive and negative sequence harmonics and (b) for zero sequence harmonics.

The aim of the discussion conducted below is to design a relation determining the rms value of the $i_{c C}$ component of the current of the $C$ capacitor. Figure 6 shows the harmonic spectrum of the current component $i_{c C}$ expressed in the p.u. system in the LCL circuit in a system with two inverters.

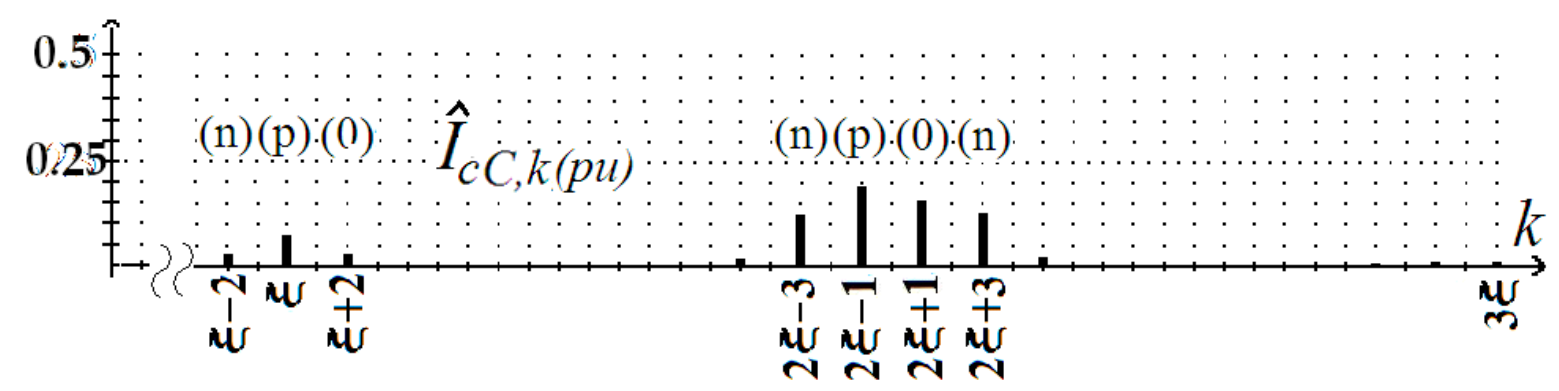

Figure 6. Harmonics spectrum of current component $i_{c C}\left(M=0.9, L_{-} 1=76 \mu \mathrm{H}, L_{-2}=84 \mu \mathrm{H}\right)$, where $(\mathrm{n}),(\mathrm{p}),(0)$ denote negative, positive and zero sequence harmonics, respectively (for the same sequence of $u_{g 1,1}^{\prime}, u_{g 2,1}^{\prime}, u_{g 3,1}^{\prime}$ voltages and wave carriers).

The amplitude of harmonic $\hat{I}_{c C, k(p u)}$ is described by the relation: $\hat{I}_{c C, k(p u)}=\hat{I}_{c C, k} / I_{b}$. Base current $I_{b}$ is defined by relation $I_{b}=U_{d c} /\left(8 L f_{C}\right)$ [17]. $I_{b}$ is equal to the maximum output current ripple of each branch of the inverter for $u_{c i}=0$, where $i \epsilon\{1,2,3\}$.

The capacitance $C$ and inductors $L_{-1}$ and $L_{-2}$ of $L$ nominal inductance are selected so that the resonant frequency $f_{\text {res }}$ of the LCL system is given by 


$$
f_{\text {res }}=\frac{1}{2 \pi} \sqrt{\frac{L+2\left(L_{l q}+L_{g}^{\prime}\right)}{L\left(L_{l q}+L_{g}^{\prime}\right) C}}
$$

and approximately satisfies the conditions given in $[28,29]$

$$
10 f_{g}<f_{\text {res }}<0.5 f_{C}
$$

where $f_{g}=\omega_{g} /(2 \pi)$ and $f_{C}$ is the carrier frequency. When the value of the inductances of the chokes $L_{-} 1$ and $L_{-} 2$ are within the tolerance of $5 \%$, then the value of $f_{\text {res }}$ determined by Equation (15) yields an error lower than $\pm 2.5 \%$.

The lower limit of the condition in Equation (16) was adopted for an LCL filter in a three-phase grid-connected inverter [28]. For LCL filters used in an PAPF, the lower limit given in [6] depends on the maximum value of the compensated harmonic order $\left(k_{\max }\right)$ and is $4 k_{\max } f_{g}$, so is usually greater than $10 f_{g}$.

$$
4 k_{\max } f_{g}<f_{\text {res }}<0.5 f_{C}
$$

Further analysis will repeatedly be based on simplifications resulting from a sufficiently high value of $\xi=f_{C} / f_{g}$. The required value $\xi=8 k_{\max }$ can be determined from the limit values of $f_{\text {res }}$. If we assume that the three-wire three-phase PAPF compensates only the fifth and lower harmonics, then the least favorable value of $f_{C} / f_{g}=40$ is obtained (from the point of view of analysis accuracy). This value is in accordance with the assumption in Section $3\left(f_{C} / f_{g} \geq 40\right)$.

In systems consisting of parallel interleaved inverters the upper limit of $f_{\text {res }}$ given by Equations (16) and (17) can have higher values [30]. This is due to the fact that for $L_{-} 1=L_{-2}$ the minimum frequency of the higher harmonics of the LCL filter capacitor current is approximately equal to the double frequency of the carrier harmonic $f_{C}$ (Figure 6). On the other hand, with the limited accuracy of the $L_{-} 1$ and $L_{-} 2$ inductances, a harmonic of frequency $f_{C}$ may appear in the current of the capacitor $C$. As can be seen from calculations supported by simulation research, when the values of $L_{-} 1$ and $L_{-2}$ are held to within $5 \%$, the value of amplitude $\hat{I}_{c C, \xi}$ for modulation depth $M=0.9$ is close to $45 \%$ of the value of amplitude $\hat{I}_{c C, 2 \xi-1}$. The influence of the harmonic of the carrier frequency on the risk of creating a current resonance in the LCL filter is high. Due to the above, further analysis of the effective current in the LCL filter capacitor assumes that Equation (17) is fulfilled. Meeting the upper limit of the inequality of Equation (17) is equivalent to meeting the $f_{C} / f_{\text {res }}>2$ condition.

The coefficients of current components with a positive and negative harmonic sequence $I_{c C, k}^{(p, n)}$ and with zero harmonic sequence $I_{c C, k}^{(0)}$ can be determined on the basis of the equivalent circuit of the diagrams shown in Figure 5a,b respectively. Resulting from Equations (3a), (3b) (4a) and (4b) the harmonics of voltages $u_{o_{-} 1, k}$ and $u_{0_{-} 2, k}$ for $m=2,4,6 \ldots$ are equal. The time waveform current harmonic $i_{c C, i, k}$ is described by:

$$
i_{c C, i, k}=I_{c C, k} \cos \left[k \omega_{g} t+m \theta_{C i_{-} 1}+n \theta_{g i}^{\prime}+\varphi_{c, k}\right] \text { for } m=2,4,6 \ldots
$$

where $\varphi_{c, k}$ is the phase shift for the $k$-th harmonic of the current $i_{c C, k}$ forced by the circuit's impedance.

In order to determine the relationships describing the harmonic coefficients $I_{C C, k}$, it is convenient to present the current $i_{c C, k}$, voltages $u_{o_{-} 1, k}$ and $u_{0_{-} 2, k}$ and impedances in circuits in complex forms $\underline{I}_{c C, k}=I_{c C, k} e^{j\left(\pi / 2+m \theta_{C 1_{1} 1}+\varphi_{c, k}\right)}, \underline{U}_{o_{-} 1, k}=U_{o_{-} 1, k} e^{j\left(\pi / 2+m \theta_{C 1 \_}+n \theta_{g}^{\prime}\right)}, \underline{U}_{o_{-} 2, k}=U_{o_{-}, k} e^{j\left(\pi / 2+m \theta_{C 1 \_}+n \theta_{g}^{\prime}\right)}$, $\underline{X}_{1}=j k \omega_{g} L_{-} 1, \underline{X}_{2}=j k \omega_{g} L_{-} 2, \underline{X}_{3}=j k \omega_{g}\left(L_{q l}+L_{g}^{\prime}\right)$, and $\underline{X}_{c}=1 /\left(j k \omega_{g} C\right)$, and then apply the Thevenin method. After a few transformations we get

$$
I_{c C, k}^{(p, n)}=\frac{U_{o_{-} 1, L_{-} L_{2}+U_{o \_}, k} L_{-} 1}{L_{-2}+L_{-} 1} \frac{L_{l q}+L_{g}^{\prime}}{L_{e q}+L_{l q}+L_{g}^{\prime}} \frac{1}{Z_{c, k}} \text { for } m \pm n= \pm 1, \pm 2, \pm 4, \pm 5, \pm 7 \ldots
$$




$$
I_{c C, k}^{(0)}=\frac{U_{0 \_1, k} L_{\_}+U_{0 \_2, k} L_{\_} 1}{L_{\_} 2+L_{-} 1} \frac{1}{Z_{c, k}^{(0)}} \text { for } m \pm n=0, \pm 3, \pm 6 \ldots
$$

where $L_{e q}=L_{-1} L_{-2} /\left(L_{-} 1+L_{-2}\right)$ while $Z_{c, k}$ and $Z_{c, k}^{(0)}$ are the equivalent impedances of the circuit for positive and negative sequence harmonics $\left(Z_{c, k}\right)$ and zero sequence harmonics $\left(Z_{c, k}^{(0)}\right)$, respectively, given by

$$
\begin{gathered}
Z_{c, k}=\sqrt{\left[k \omega_{g} \frac{\left(L_{l q}+L_{g}^{\prime}\right) L_{e q}}{L_{e q}+L_{l q}+L_{g}^{\prime}}-\frac{1}{k \omega_{g} C}\right]^{2}+R_{d}^{2}} \\
Z_{c, k}^{(0)}=\sqrt{\left[k \omega_{g} L_{e q}-1 /\left(k \omega_{g} C\right)\right]^{2}+R_{d}^{2}}
\end{gathered}
$$

Equations (19a) and (19b) is true for systems in which voltages $u_{g 1,1}^{\prime}, u_{g 2,1}^{\prime}$, and $u_{g 3,1}^{\prime}$ and the voltages $u_{T 1 \_1,1}, u_{T 2 \_1,1}$, and $u_{T 3 \_1,1}$ form the same or the opposite sequence. In the first case, the conditions in the formulae are related to the sum of $m+n$, in the second case to the difference of $m-n$.

For $m \geq 2, \xi \geq 40$, and $|n| \leq 3$, the approximation $m \xi \approx m \xi+n$ has an error of no more than $3.75 \%$. After taking the approximation $k=m \xi$ and taking into account in Equations (20a) and (20b) the relations given in Equations (9) and (15), as well as $\xi=f_{C} / f_{g}$, we obtain

$$
\begin{gathered}
Z_{c, m \xi}=m \omega_{C} \frac{\left(L_{l q}+L_{g}^{\prime}\right) L_{e q}}{L_{e q}+L_{l q}+L_{g}^{\prime}} \sqrt{\left[1-\frac{f_{r e s}^{2}}{m^{2} f_{C}^{2}}\right]^{2}+\left(\frac{2 \xi_{p} f_{r e s}}{m f_{C}}\right)^{2}} \\
Z_{c, m \xi}^{(0)}=m \omega_{C} L_{e q} \sqrt{\left[1-\frac{f_{r e s}^{2}}{m^{2} f_{C}^{2}}\left(1-\frac{L_{e q}}{L_{\Sigma}}\right)\right]^{2}+\left[\frac{2 \xi_{p} f_{r e s}}{m f_{C}}\left(1-\frac{L_{e q}}{L_{\Sigma}}\right)\right]^{2}}
\end{gathered}
$$

where $L_{\Sigma}=L_{e q}+L_{l q}+L_{g}^{\prime}$.

Reference [6] recommends for a PAPF equal inductances on the grid and converter-side to produce the lowest resonance frequency, while in [28] it is recommended for the three-phase active rectifier that the ratio between converter-side and grid-side inductances is in the range of 3-7. If we assume the limit values $L_{\Sigma} / L_{e q}=2, m=2, f_{C} / f_{\text {res }}=2, \zeta_{p}=0$, then the value of the square root in Equation (21b) maximally deviates from unity and reaches the value of 0.97 . The value of the square root in Equation (21a) maximally deviates from the unity for $m=2, f_{C} / f_{\text {res }}=2, \zeta_{p}=0$ and is equal to 0.94 . Therefore, for $m \geq 2$, it is possible to determine $Z_{c, m \xi}$ and $Z_{c, m \xi}^{(0)}$ with errors not exceeding $6 \%$ and $3 \%$, respectively, using the following dependencies:

$$
\begin{gathered}
Z_{c, m \xi}=m \omega_{C} \frac{\left(L_{l q}+L_{g}^{\prime}\right) L_{e q}}{L_{e q}+L_{l q}+L_{g}^{\prime}} \\
Z_{c, m \xi}^{(0)}=m \omega_{c} L_{e q}
\end{gathered}
$$

The harmonic spectrum presented in Figure 6 for an PAPF with two inverters shows that the value $\hat{I}_{c C}$ depends on harmonics whose order is close to the value of $2 \xi$. To calculate the rms value of current $I_{c C, 2 \xi(r m s)}^{(p, n)}$, we need to find the square root of the sum of its squares for $n=-3,-1,3$. Using Equation (19a) and assuming $U_{0_{-} 2,2 \xi+n}=U_{o_{-} 1,2 \xi+n}$ we obtain the relation, without introducing a significant error, for the value of $\xi \geq 40$ :

$$
I_{c C, 2 \xi(r m s)}^{(p, n)}=\frac{\sqrt{2}}{2 Z_{c, 2 \xi}} \frac{L_{l q}+L_{g}^{\prime}}{L_{e q}+L_{l q}+L_{g}^{\prime} g} \sqrt{\sum_{n=-3,-1,3} U_{o_{-} 1,2 \xi+n}^{2}}
$$


If $L_{-2} \neq L_{-1}$ then $I_{\mathcal{C C}, \xi}$ is different than zero. By substituting $U_{o 2, \xi}=-U_{o_{-} 1, \xi}$ into Equation (19a) we get

$$
I_{c C, \xi(r m s)}^{(p, n)}=\frac{\sqrt{2}}{2 Z_{c, \xi}}\left|\frac{L_{-}-L_{-1}}{L_{-2}+L_{-1}}\right| \frac{L_{l q}+L_{g}^{\prime}}{L_{e q}+L_{l q}+L_{g}^{\prime}} U_{o_{-} 1, \xi}
$$

By substituting $U_{0_{-} 2,2 \xi+1}=U_{o_{-} 1,2 \xi+1}$ into Equation (19b), the rms value of current $I_{c C}^{(0)}$ is given by

$$
I_{c C, 2 \xi+1(r m s)}^{(0)}=U_{o_{-} 1,2 \xi+1} /\left(\sqrt{2} Z_{c, 2 \xi}^{(0)}\right)
$$

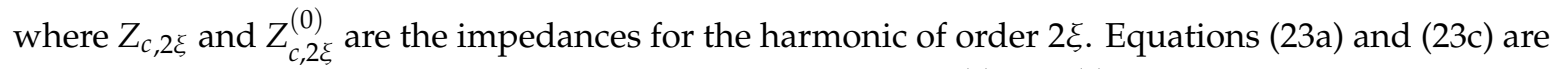
based on the assumption that $Z_{c, 2 \xi} \approx Z_{c, 2 \xi-1} \approx Z_{c, 2 \xi \pm 3}$ and $Z_{c, 2 \xi}^{(0)} \approx Z_{c, 2 \xi+1}^{(0)}$. After substituting $m=2$ and $\omega_{C} / \omega_{g}=\xi$ into Equations (2) and (4a), we obtain for each pair of corresponding branches of the inverters:

$$
U_{D_{-} 1,2 \xi+n}=-\frac{U_{d c}}{\pi} \frac{J_{n}([1+n /(2 \xi)] \pi M)}{1+n /(2 \xi)} \sin \left(n \frac{\pi}{2}\right)
$$

The harmonic spectrum justifies limiting the scope of the order of harmonics included in Equation (23a) to the range $(2 \xi-3,2 \xi+3)$. Since for $n=-3,-1,1,3$, the $(2 \xi>>n)$ inequality applies, Equation (24) can be simplified to

$$
U_{o_{-} 1,2 \xi+n}=-\left(U_{d c} / \pi\right) J_{n}(\pi M) \sin (n \pi / 2)
$$

After introducing Equations (22a) and (25) into Equation (23a) we get

$$
I_{c C, 2 \xi(r m s)}^{(p, n)}=\frac{\sqrt{2} U_{d c}}{4 \pi \omega_{C} L_{e q}} \sqrt{J_{1}^{2}(\pi M)+2 J_{3}^{2}(\pi M)}
$$

Similarly, we get

$$
I_{c C, 2 \xi+1(r m s)}^{(0)}=\sqrt{2} U_{d c} J_{1}(\pi M) /\left(4 \pi \omega_{C} L_{e q}\right)
$$

Because Equation (22b) can be used for $m \geq 2$, the impedance $Z_{c, \xi}$ in Equation (23b) specified for $m=1$ has been replaced by another, simplified relation (Equation (21a)) to give

$$
I_{c C, \xi(r m s)}^{(p, n)}=\frac{\sqrt{2} U_{d c}}{\pi \omega_{C} L_{e q}}\left|\frac{L_{-2}-L_{-} 1}{L_{-} 2+L_{-} 1}\right| \frac{1}{1-\left(f_{r e s} / f_{C}\right)^{2}} J_{0}\left(\frac{\pi M}{2}\right)
$$

To calculate the rms value $I_{\mathcal{C}, r m s}$ for the inductance with a non-zero value tolerance, we need to find the square root:

$$
I_{c C, r m s}=\frac{U_{d c}}{2 \pi \omega_{C} L_{e q}} \sqrt{J_{1}^{2}(\pi M)+J_{3}^{2}(\pi M)+8\left[\frac{(\% \Delta L)}{100} \frac{J_{0}(\pi M / 2)}{1-\left(f_{r e s} / f_{C}\right)^{2}}\right]^{2}}
$$

where $(\% \Delta \mathrm{L})$ is the tolerance of the inductance of chokes $L_{-1}$ and $L_{-2}$. If $L_{-1}=L_{-} 2=L_{\text {, then }}$ rms value $I_{c C, r m s}$ can be calculated from the equation:

$$
I_{c C, r m s} \approx \frac{U_{d c}}{\pi \omega_{C} L} \sqrt{J_{1}^{2}(\pi M)+J_{3}^{2}(\pi M)}
$$

An equation that describes the rms of capacitor $C$ current takes into account all the above components and takes the following form:

$$
I_{c, r m s}=\sqrt{I_{c G L, r m s}^{2}+I_{c C, r m s}^{2}}
$$


The values of the functions used in Equations (29a) and (29b) are presented in Table 4.

Table 4. The values of functions used in formulae.

\begin{tabular}{|c|c|c|c|c|c|c|c|c|c|c|c|}
\hline Functions & & & & & & Values & & & & & \\
\hline \multirow{2}{*}{$M$} & 0.00 & 0.1 & 0.2 & 0.3 & 0.4 & 0.5 & 0.6 & 0.7 & 0.8 & 0.9 & \multirow{2}{*}{1} \\
\hline & 0.05 & 0.15 & 0.25 & 0.35 & 0.45 & 0.55 & 0.65 & 0.75 & 0.85 & 0.95 & \\
\hline \multirow{2}{*}{$J_{0}(\pi M / 2)$} & 1.000 & 0.994 & 0.975 & 0.945 & 0.904 & 0.852 & 0.790 & 0.720 & 0.643 & 0.559 & \multirow{2}{*}{0.472} \\
\hline & 0.998 & 0.986 & 0.962 & 0.926 & 0.879 & 0.822 & 0.756 & 0.682 & 0.602 & 0.516 & \\
\hline \multirow{2}{*}{$\sqrt{J_{1}^{2}(\pi M)+J_{3}^{2}(\pi M)}$} & 0.000 & 0.155 & 0.299 & 0.421 & 0.514 & 0.571 & 0.592 & 0.579 & 0.540 & 0.487 & \multirow{2}{*}{0.438} \\
\hline & 0.078 & 0.229 & 0.363 & 0.471 & 0.547 & 0.586 & 0.589 & 0.562 & 0.515 & 0.461 & \\
\hline
\end{tabular}

\section{The Ripple Current in Dc-Link Capacitors}

Because the simulation research showed a small influence of $L_{-1}$ and $L_{-2}$ choke inductance asymmetry on the rms value of the total current in dc-link capacitors forced by the ripple of output currents of the inverter branch, the analysis concerning the rms value of this current will be carried out with the assumption of $L_{-1}=L_{-2}=L$.

The harmonics of current $i_{v}$ forced by the harmonics of current flowing in the ac-circuit for orders typical of $\operatorname{PAPF}(5,7,11,13 \ldots)$ can be determined on the basis of the analysis given in $[8,31]$.

$$
I_{v, 3 n(r m s)}=\frac{3}{2} \frac{\hat{U}_{g, 1}^{\prime}}{U_{d c}} \sqrt{\begin{array}{l}
\left(\lambda_{3 n-1} I_{L, 3 n-1(r m s)}^{\prime} \sin \varphi_{L, 3 n-1}^{\prime}+\lambda_{3 n+1} I_{L, 3 n+1(r m s)}^{\prime} \sin \varphi_{L, 3 n+1}^{\prime}\right)^{2}+ \\
\left(\lambda_{3 n-1} I_{L, 3 n-1(r m s)}^{\prime} \cos \varphi_{L, 3 n-1}^{\prime}+\lambda_{3 n+1} I_{L, 3 n+1(r m s)}^{\prime} \cos \varphi_{L, 3 n+1}^{\prime}\right)^{2}
\end{array}}
$$

where $I_{L, k(r m s)}^{\prime}$ is the rms value of the compensated $k$-th harmonic load current, brought to the primary side of the transformer.

If the PAPF does not compensate the reactive component of the fundamental harmonic, the share of harmonics of current $i_{v}$ forced by the ripple currents in the inductors, $L_{-} 1$ and $L_{-2}$ increase significantly. The current denoted in Figure $1 \mathrm{a}$ as $i_{c p}$ is half of the sum of currents of LCL filter capacitors flowing in dc-circuits.

$$
i_{c p}=\left(i_{c 1}+i_{c 2}+i_{c 3}\right) / 2
$$

The equation for the current in the dc-circuit is as follows:

$$
i_{d c}=i_{v}-i_{c p}
$$

where $i_{v}$ is the current consumed by two inverters $\left(i_{v}=i_{v 1}+i_{v 2}\right)$ and $i_{d c}$ is the capacitor $C_{d c}$ current. The following relation can be used to determine $i_{v}$ current:

$$
i_{v}=s_{1 \_} i_{01 \_1}+s_{2 \_} i_{02 \_}+s_{3 \_} i_{03 \_}+s_{1 \_} i_{01 \_2}+s_{2 \_} i_{02 \_}+s_{3 \_} i_{03 \_} 2
$$

where $s_{i j}$ denotes the switching function for the $i$-th branch of the $j$-th inverter.

Below, the harmonic coefficients of the dc-link current component $i_{v C}$, forced only by the carrier and sideband harmonics of the inverter branch output currents will be determined, with the absence of fundamental and baseband harmonics in this current. Thus, the following analysis applies to harmonic currents in the inductors $L_{-} 1$ and $L_{-2}$, for which the carrier index variable meets the assumption of $m \geq 1$.

\subsection{Current $i_{c p}$}

The harmonics of voltages $u_{o i j}$ of $2 \xi+1,2 \xi-5,4 \xi-1,4 \xi-7,4 \xi+5$ order in the three-phase system make zero sequence components. If the relation of Equation (17) is fulfilled, $m \geq 1, \xi \geq 40$ 
and $|n| \leq 3$ then impedance $Z_{c, m \xi+n}^{(0)}$ for the mentioned harmonics is of inductive nature and increases significantly as its order increases. Thus, the order of the dominating harmonic is $2 \xi+1$. The amplitude of the harmonics sum of the currents $i_{c C 1, k}+i_{c C 2, k}+i_{c C 3, k}$ of this order, assuming that $Z_{c, 2 \xi+1}^{(0)} \approx Z_{c, 2 \xi^{\prime}}^{(0)}$ $\varphi_{c, 2 \xi+1}^{(0)} \approx \varphi_{c, 2 \xi^{\prime}}^{(0)}$ is triple the amplitude $\hat{I}_{c, 2 \xi+1}$. The harmonic coefficient $I_{c, 2 \xi+1}$ can be determined from Equations (18), (19b) and (25) for $m=2$ and $n=1$ taking into account the equality $m \theta_{C i_{-} 1}+n \theta_{i}^{\prime}=m \theta_{C 1_{-} 1}$ is true for $i=1,2,3$ if $m+n=3$. The time waveform of the current harmonic $i_{c p, 2 \xi+1}$ is described by

$$
i_{c p, 2 \xi+1}=I_{c p, 2 \xi+1} \cos \left[(2 \xi+1) \omega_{g} t+m \theta_{C 1 \_1}+\varphi_{c, 2 \xi}^{(0)}\right]
$$

where coefficient $I_{c p, 2 \xi+1}$ and phase angle $\varphi_{c, 2 \xi}^{(0)}$ are described by the following relations:

$$
\begin{gathered}
I_{c p, 2 \xi+1}=-3 U_{d c} J_{1}(\pi M) /\left(2 \pi Z_{c, 2 \xi}^{(0)}\right) \\
\varphi_{c, 2 \xi}^{(0)}=-\operatorname{arctg}\left\{\left[\omega_{C} L-1 /\left(2 \omega_{C} C\right)\right] / R_{d}\right\}
\end{gathered}
$$

For real values of parameters $\xi, L, C$, and $R_{d}$ in a power system with an PAPF, phase angle $\varphi_{c, 2 \xi}^{(0)} \approx-\pi / 2 \mathrm{rad}$.

\subsection{Dc-Link Capacitor Current Component $i_{d c C}$}

The dc-link component of the capacitor current and the $i_{v 1}, i_{v 2}, i_{v}$, and $i_{c p}$ current components forced by the ripple of the output currents of inverter branches will be denoted $i_{d c C}, i_{v 1 C}, i_{v 2 C}, i_{v C}$ and $i_{c p C}$, respectively. Figure 7 shows the harmonics spectrum of currents $i_{v 1 C}, i_{v 2 C}, i_{v C}, i_{c p C}$ and $i_{d c C}$ determined using the numerical model shown in Figure $1 \mathrm{a}$ for the modulation factor $M=0.9$

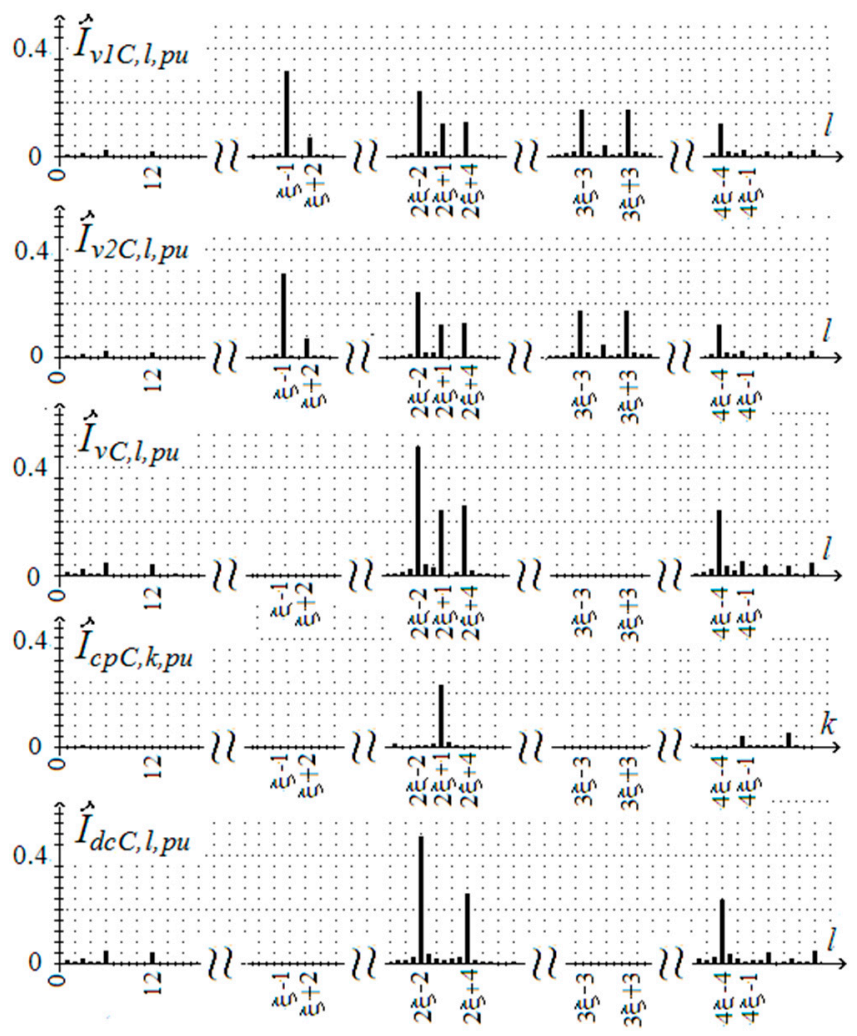

Figure 7. Harmonics spectrum of currents $i_{v 1 C}, i_{v 2 C}, i_{v C}, i_{c p C}$ and $i_{d c C}$ in the system without load for the same sequence $u_{g 1,1}^{\prime}, u_{g 2,1}^{\prime}$ and $u_{g 3,1}^{\prime}$ voltages and wave carriers $(M=0.9)$. 
Assuming sinusoidal voltages $u_{c 1}, u_{c 2}$, and $u_{c 3}$, the switching function for the $i$-th branch of the $j$-th inverter can be expressed in Fourier form by

$$
s_{i-j}=0.5+\sum_{n_{s}=1}^{\infty} S_{0 n_{s}} \cos \left(n_{s} \omega_{g} t+n_{s} \theta^{\prime}{ }_{g i}\right)+\sum_{m_{s}=1}^{\infty} \sum_{n_{s}=-\infty}^{\infty} S_{m_{s} n_{s}} \cos \left[\left(m_{s} \xi+n_{s}\right) \omega_{g} t+m_{s} \theta_{C i_{-} j}+n_{s} \theta^{\prime}{ }_{g i}\right]
$$

where $S_{m_{s} n_{s}}=F_{m_{s} n_{s}} / U_{d c}$.

The order of harmonic switching function is $k_{s}=m_{s} \xi+n_{s}$. The output current of the $i$-th branch of the $j$-th inverter also includes the harmonics of the same order. The individual harmonics of the switching function and the currents in the three-phase system make positive, negative, or zero sequence depending on the respective algebraic sums $m_{s} \pm n_{s}$ or $m \pm n$ according to the equations given in Section 4.3. Figure 8 shows the harmonics spectrum of switching function $s_{i j}$.

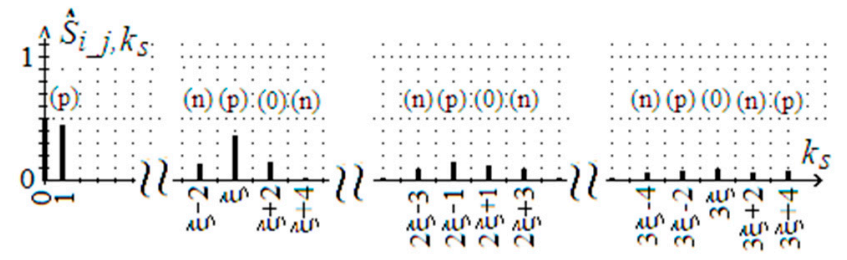

Figure 8. Harmonics spectrum of switching function $s_{i_{-} j}$ for the same sequence $u_{g 1,1^{\prime}}^{\prime} u_{g 2,1}^{\prime}$, and $u_{g 3,1}^{\prime}$ voltages and wave carriers $(\mathrm{M}=0.9)$.

According to the considerations made in [31], certain pairs of current harmonic and switching function harmonic can force the harmonic of current $i_{v}$ to be of corresponding order, if certain conditions are met.

For large values of $\xi\left(\xi \geq 40\right.$ was assumed), harmonics coefficients of $S_{k_{s}}$ can be described by the equation

$$
S_{k_{s}}=\left\{\begin{array}{lr}
1 / 2 & \text { for } \begin{array}{r}
m_{s}=0 \\
n_{s}=0
\end{array} \text { and } \\
\frac{2 \xi}{n_{s} \pi} J_{n_{s}}\left(\frac{n_{s} \pi M}{2 \xi}\right) \sin \left(\frac{n_{s} \pi}{2}\right) & \text { for } \begin{array}{r}
m_{S}=0 \\
n_{S} \neq 0
\end{array} \text { and } \\
\frac{2}{m_{s} \pi} J_{n_{s}}\left(\frac{m_{s} \pi M}{2}\right) \sin \left(\frac{\pi\left[m_{s}+n_{s}\right]}{2}\right) & \text { for } m_{s} \neq 0
\end{array}\right.
$$

For $m_{s} \neq 0$ and $\xi \geq 40$ the approximation of $m_{s}+n_{s} / \xi \approx m_{s}$ was adopted. Similarly for $\xi \geq 40$ the approximation $(2 \xi / \pi) J_{1}(\pi M / 2 \xi) \approx M / 2$ is true, which results in

$$
S_{01} \approx M / 2
$$

Labels $S_{k_{s}}$ and $S_{m_{s} n_{s}}$ are the same $\left(S_{k_{s}}=S_{m_{s} n_{s}}\right)$.

The source of the inductor $L$ current ripple is the carrier and sideband harmonics forced by the inverter of the PAPF. The first component (for harmonics of any sequence) of the $i_{o k}$ flows between the outputs of the branches of the same phase of the PAPF inverters. This component is sourced from harmonics of the difference of the output voltages of the corresponding pair of inverter branches ( $m=1,3 \ldots, n= \pm 2, \pm 4 \ldots$ ). The second (for zero sequence harmonics) and third (for positive and negative sequence harmonics) components of the inductor ripple current contains sideband harmonics $(m=2,4 \ldots, n= \pm 1, \pm 3 \ldots)$.

The time waveform of the output current harmonic of the $i$-th branch of the $j$-th inverter is given by

$$
i_{o, i_{-} j, k}=I_{o, k} \cos \left[(m \xi+n) \omega_{g} t+m \theta_{C i_{-} j}+n \theta^{\prime}{ }_{g i}+\varphi_{0, m \xi+n}\right]
$$


For $m \neq 0$ (analysis pertains to the impact of ripple currents in the inductors of equal $L_{-1}$ and $L_{-} 2$ values on the rms value of capacitor $C_{d c}$ current), harmonic coefficients of three components of currents $i_{o, i} i_{j}$ are given by

$$
\begin{aligned}
& I_{o, k}=\frac{2 U_{d c}}{m^{2} \pi \omega_{C} L} J_{n}\left(\frac{m \pi M}{2}\right) \sin \frac{\pi(m+n)}{2} \text { for } \quad m=1,3,5 \ldots \\
& I_{o, k}=\frac{U_{d c}}{m \pi Z_{c, m \xi}^{(0)}} J_{n}\left(\frac{m \pi M}{2}\right) \sin \frac{\pi(m+n)}{2} \text { for } \begin{array}{c}
m=2,4,6 \ldots \\
m \pm n= \pm 3, \pm 6, \pm 9 \ldots
\end{array} \\
& I_{o, k}=\frac{2 U_{d c}}{m \pi Z_{o, m \xi}} J_{n}\left(\frac{m \pi M}{2}\right) \sin \frac{\pi(m+n)}{2} \text { for } \begin{array}{cc}
m & =2,4,6 \ldots \\
m \pm n & = \pm 1, \pm 2, \pm 4, \pm 5, \pm 7 \ldots
\end{array}
\end{aligned}
$$

respectively, where $Z_{c, m \xi}^{(0)}$ is given by Equation (22b) for $L_{e q}=L / 2, Z_{o, m \xi}=Z_{o, k}$ for $k=m \xi$. Equations (42a) and (42b) result from the diagram shown in Figure $5 b$, and the fulfilment of the conditions for their applicability means $I_{c \mathcal{C}, k}=0$ and $I_{0, k}=I_{c C, k}^{(0)} / 2$, respectively.

Impedance $Z_{o, m \xi}$ (for $\left.m=2,4,6 \ldots\right)$, seen from the output terminal of the VSI branch $\left(Z_{o, m \xi}=\right.$ $\left.\left|U_{o_{1} 1, k} / I_{o_{-} 1, k}\right|=\left|U_{o_{-} 2, k} / I_{o_{-} 2, k}\right|\right)$, has been determined considering the equivalent circuit shown in Figure $5 \mathrm{a}$ assuming $L_{-} 1=L_{-} 2=L$. Due to $m=2,4,6 \ldots$ and the equations $U_{o_{-} 1, k}=U_{o_{-} 2, k}$ and $I_{O_{-} 1, k}=I_{O_{-} 2, k}$ being met, the impedance $Z_{o, m \xi}$ is equal to double the value of the impedance of the circuit containing the elements between the shorted "out_1" and "out_2" outputs and the point with potential "0".

$$
Z_{o, k}=2 k \omega_{g} \sqrt{\frac{\left[L_{\Sigma}-0.5 k^{2} \omega_{g}^{2} C L\left(L_{l q}+L_{g}^{\prime}\right)\right]^{2}+\left(k \omega_{g} C R_{d} L_{\Sigma}\right)^{2}}{\left[1-k^{2} \omega_{g}^{2} C\left(L_{l q}+L_{g}^{\prime}\right)\right]^{2}+\left(k \omega_{g} C R_{d}\right)^{2}}}
$$

where $L_{\Sigma}=L / 2+L_{l q}+L_{g}^{\prime}$. Having included the relations $k=m \xi$, $\xi=f_{C} / f_{g}$, and Equations (9) and (15) in Equation (43) we get

$$
Z_{o, m \xi}=m \omega_{c} L \sqrt{\left(\frac{2 L_{\Sigma}}{L}\right)^{2} \frac{\left(1-m^{2} f_{C}^{2} / f_{r e s}^{2}\right)^{2}+\left(2 \xi_{p} m f_{C} / f_{r e s}\right)^{2}}{\left(1-\frac{2 L_{\Sigma}}{L} m^{2} f_{C}^{2} / f_{r e s}^{2}\right)^{2}+\left(2 \xi_{p} m f_{C} / f_{r e s}\right)^{2}}}
$$

If we take the limit values $2 L_{\Sigma} / L=2, m=2, f_{C} / f_{\text {res }}=2, \zeta_{p}=0$, then the value of the square root in Equation (44) deviates maximally from unity and reaches the value of 0.97 . This means that on the basis of the relation in Equation (45), we can determine $Z_{o, m \xi}$ with an error of no more than $3 \%$.

$$
Z_{o, m \xi}=m \omega_{C} L
$$

The form of Equation (45) shows the inductive impedance character, and, therefore, $\varphi_{0, m \xi} \approx$ $-\pi / 2 \mathrm{rad}$. Similarly the inductive character of impedances $Z_{c, m \xi}^{(0)}$ and $\varphi_{c, m \xi}^{(0)} \approx-\pi / 2$ rad results from Equation (22b).

As can be seen from Equation (34), the dc-link current is the result of superposition of all six switch branches currents. Each dc-link current $i_{v l}$, harmonic of the $l$ order can be a product of many harmonic pairs of functions switching any order of $k_{s}$ and output currents of branches of any order of $k$ but for which $\left|k_{s} \pm k\right|$ is constant $[10,31]$.

$$
i_{v, l}=\sum_{k_{s}, k} i_{v, k_{s} \pm k}^{(c o m p)} \text { for }\left|k_{s} \pm k\right|=\text { const }
$$

where $i_{v, k_{s} \pm k}^{(\text {comp })}$ is the component of the $i_{v, l}$ harmonic. In order to determine one harmonic current component $i_{v, k_{s} \pm k}^{(c o m p)}$, we can substitute into Equation (34) a harmonic of output current $i_{0, i_{-} j, k}$ of $k$ 
order which is described by Equation (41) and a harmonic of switching function $s_{i_{-j}, k_{s}}$ of $k_{s}$ order described below:

$$
s_{i_{-j}, k_{s}}=\left\{\begin{array}{cccc}
1 / 2 & \text { for } & m_{s}=0 & \text { and } \\
S_{m_{s} n_{s}} \cos \left[\left(m_{s} \xi+n_{s}\right) \omega_{g} t+m_{s} \theta_{C i_{-j} j}+n_{s} \theta^{\prime}{ }_{g i}\right] & & \text { for } & m_{s} \neq 0
\end{array}\right.
$$

Then we obtain the equations describing $i_{v, k}^{(\text {comp })}, i_{v, k_{s}+k}^{(\text {comp })}$ and $i_{v, k_{s}-k}^{(c o m p)}$ in the following forms:

$$
\begin{gathered}
i_{v, k}^{(c o m p)}=\frac{1}{2} I_{o, k} \sum_{j=1,2} \sum_{i=1,2,3} \cos \left[(m \xi+n) \omega_{g} t+m \theta_{C i_{-} j}+n \theta_{g i}^{\prime}+\varphi_{0, m \xi+n}\right] \\
i_{v, k_{s} \pm k}^{(c o m p)}=S_{k_{s}} I_{o, k} \sum_{j=1,2} \sum_{i=1,2,3}\left\{\cos \left[\left(m_{s} \xi+n_{s}\right) \omega_{g} t+m_{s} \theta_{C i_{-} j}+n_{s} \theta_{g i}^{\prime}\right] \cos \left[(m \xi+n) \omega_{g} t+m \theta_{C i_{-} j}+n \theta_{g i}^{\prime}+\varphi_{0, m \xi+n}\right]\right\}
\end{gathered}
$$

where the phase angles fulfill the following relations: $\theta_{C 2-1}=\theta_{C 1-1} \pm 2 \pi / 3, \theta_{C 3-1}=\theta_{C 1-1} \mp 2 \pi / 3$, $\theta_{C i-2}=\theta_{C i-1}+\pi($ for $i=1,2,3), \theta_{g 1}^{\prime}=0, \theta_{g 2}^{\prime}=-2 \pi / 3, \theta_{g 3}^{\prime}=2 \pi / 3$. After considering the trigonometric identity $\cos \alpha \cos \beta=0.5[\cos (\alpha+\beta)+\cos (\alpha-\beta)]$ and the equality $x \theta_{C i_{-} 2}=x \theta_{C i_{-} 1}$ for even $x$ we obtain the sum of three components in the form of

$$
\begin{gathered}
i_{v, k}^{(c o m p)}=\frac{1+(-1)^{m}}{2} I_{o, k} \sum_{i=1,2,3} \cos \left[(m \xi+n) \omega_{g} t+m \theta_{C 1 \_1}+(m \pm n) \theta_{g i}^{\prime}+\varphi_{o, m \xi+n}\right] \\
i_{v, k_{s}+k}^{(c o m p)}=\frac{1+(-1)^{m_{s}+m}}{2} S_{k_{s}} I_{o, k} \sum_{i=1,2,3} \cos \left\{\left[\left(m_{s}+m\right) \xi+\left(n_{s}+n\right)\right] \omega_{g} t+\left(m_{s}+m\right) \theta_{C 1 \_}+\left[\left(m_{s} \pm n_{s}\right)+(m \pm n)\right] \theta_{g i}^{\prime}+\varphi_{0, m \xi+n}\right\} \\
i_{v, k_{s}-k}^{(c o m p)}=\frac{1+(-1)^{m_{s}-m}}{2} S_{k_{s}} I_{o, k} \sum_{i=1,2,3} \cos \left\{\left[\left(m_{s}-m\right) \xi+\left(n_{s}-n\right)\right] \omega_{g} t+\left(m_{s}-m\right) \theta_{C 1_{-} 1}+\left[\left(m_{s} \pm n_{s}\right)-(m \pm n)\right] \theta^{\prime}{ }_{g i}-\varphi_{0, m \xi+n}\right\}
\end{gathered}
$$

Thus, harmonic current components $i_{v, k_{s} \pm k}^{(\operatorname{comp})}$ in combination with two inverters and with double three interleaved PWM carriers only occur when $m$ is even (in the first component) or the sum of $m+m_{S}$ is even (in the second component) or the difference $m_{s}-m$ is even (in the third component).

The interpretation of symbols " \pm " used in the expressions $(m \pm n)$ and $\left(m_{s} \pm n_{s}\right)$ should be that " + " is valid if the voltages $u_{g 1,1}^{\prime}, u_{g 2,1}^{\prime}, u_{g 3,1}^{\prime}$ create the same sequence as the $u_{T 1 \_1,1}, u_{T 2 \_1,1}, u_{T 3 \_1,1}$ signals, while " - " is valid for the opposite sequence.

For $m \geq 1, \xi \geq 40$, and $n= \pm 1, \pm 2, \pm 3$ we assume the approximations $m \xi+n \approx m \xi$ and $\varphi_{o, m \xi+n} \approx \varphi_{0, m \xi} \approx \varphi_{c, 2 \xi}^{(0)}$. For further considerations, the harmonic current components $i_{v, k}^{(\text {comp })}$ and $i_{v, k_{s} \pm k}^{(\text {comp })}$ will be denoted by $i_{v C, k}^{(c o m p)}$ and $i_{v C, k_{s} \pm k}^{(c o m p)}$, respectively:

$$
i_{v C, k}^{(c o m p)}=3 \frac{1+(-1)^{m}}{2} I_{0, k} \cos \left[(m \xi+n) \omega_{g} t+\phi_{0, m \xi}\right] \text { for } \begin{array}{ccc}
m_{s}=0 & \text { and } & n_{s}=0 \\
m \pm n & = & 0, \pm 3, \pm 6 \ldots
\end{array}
$$

where $\phi_{0, m \xi}=m \theta_{C 1 \_1}+\varphi_{c, m \xi}^{(0)}$.

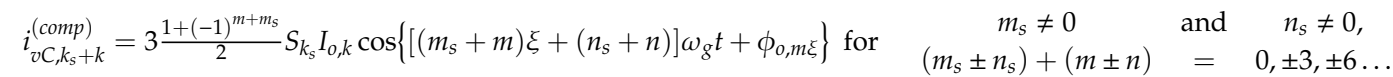

where $\phi_{o, m \xi}=\left(m_{s}+m\right) \theta_{C_{1} 1}+\varphi_{o, m \xi}$.

$$
i_{v C, k_{s}-k}^{(c o m p)}=3 \frac{1+(-1)^{m_{s}-m}}{2} S_{k_{s}} I_{o, k} \cos \left\{\left[\left(m_{s}-m\right) \xi+\left(n_{s}-n\right)\right] \omega_{g} t+\phi_{0, m \xi}\right\} \text { for } \begin{array}{ccc}
m_{s} \neq 0 & \text { and } & n_{s} \neq 0 \\
\left(m_{s} \pm n_{s}\right)-(m \pm n) & = & 0, \pm 3, \pm 6 \ldots
\end{array}
$$

where $\phi_{0, m \xi}=\left(m_{s}-m\right) \theta_{C 1 \_1}-\varphi_{o, m \xi}$. The forms of the first components of Equations (48a), (49a) and (50a) formed on the basis of a constant value of the switching function $\left(s_{00}=0.5\right)$ indicate that the harmonic coefficient $I_{v C, l}$ is proportional to the harmonic coefficient $I_{o, k}$ : 


$$
I_{v C, l}=I_{v C, k}=3 \frac{1+(-1)^{m}}{2} I_{o, k}
$$

As a product of the switching function, the harmonic coefficient of current $i_{v C, l}$ for $k_{s}>0$ can be determined as a sum of harmonic coefficients of individual components of this harmonic

$$
I_{v C, l}=3 \sum_{k_{s}, k} \frac{1+(-1)^{m_{s}+m}}{2} S_{k_{s}} I_{0, k} \text { for } k_{s}+k=\text { const }
$$

or

$$
I_{v C, l}=3 \sum_{k_{s}, k} \frac{1+(-1)^{m_{s}-m}}{2} S_{k_{s}} I_{o, k} \text { for }\left|k_{s}-k\right|=\text { const }
$$

The harmonic coefficient $I_{0,2 \xi+1}$ resulting from Equation (42b) has the form

$$
I_{0,2 \xi+1}=-\frac{U_{d c}}{2 \pi} \frac{J_{1}(\pi M)}{Z_{c, 2 \xi}^{(0)}}
$$

Based on Equations (50a) and (52), applicable for the constant component of the switching function $S_{00}=0.5$, we determine the time waveform $i_{v C, 2 \xi+1}$ :

$$
i_{v C, 2 \xi+1}=-\frac{3 U_{d c}}{2 \pi} \frac{J_{1}(\pi M)}{Z_{c, 2 \xi}^{(0)}} \cos \left([2 \xi+1] \omega_{g} t+m \theta_{C 1 \_1}+\varphi_{c, 2 \xi}^{(0)}\right)
$$

We obtain the relation describing $i_{v C, 2 \xi+1}$ identical to Equation (35). This means that this component does not load the capacitor $C_{d c}$. Similarly, it can be demonstrated that the harmonics of $4 \xi+5,4 \xi-7$ $\ldots$ order also do not load the capacitor $C_{d c}$.

According to research simulation, the order of the dominant harmonic current $i_{v}$ forced by the

\begin{tabular}{|c|c|c|c|c|c|c|c|c|}
\hline \multirow{2}{*}{$\begin{array}{c}\text { Input, Output Parameters } \\
\text { Sequence of } i_{o i_{-} j, k}\end{array}$} & \multicolumn{6}{|c|}{ Existing Conditions for Current Harmonics $i_{v, k}$} & \multicolumn{2}{|c|}{ No Harmonics } \\
\hline & (p) & $(\mathrm{n})$ & $(0)$ & (p) & (n) & $(0)$ & (0) & $(\mathrm{p}),(\mathrm{n})$ \\
\hline Sequence of $s_{i \_j, k s}$ & $(\mathrm{p})$ & $(\mathrm{n})$ & $(0)$ & (n) & $(\mathrm{p})$ & $(0)$ & $(\mathrm{p}),(\mathrm{n})$ & (0) \\
\hline \multirow{2}{*}{ Conditions } & \multicolumn{6}{|c|}{$m_{s} \pm m=-6,-4,-2,0,2,4,6 \ldots$} & \multirow{3}{*}{\multicolumn{2}{|c|}{ Do not exist }} \\
\hline & \multicolumn{3}{|c|}{$\left(m_{s} \pm n_{s}\right)-(m \pm n)=0, \pm 3, \pm 6 \ldots$} & \multicolumn{3}{|c|}{$\left(m_{s} \pm m\right)+\left(n_{s} \pm n\right)=0, \pm 3, \pm 6 \ldots$} & & \\
\hline Order of harm. of $i_{v C, k \pm k_{\mathrm{s}}}^{(c o m p)}$ & \multicolumn{3}{|c|}{$a b s\left(k-k_{s}\right)$} & \multicolumn{3}{|c|}{$k+k_{s}$} & & \\
\hline
\end{tabular}
ripple current in the inductor $L$ is $2 \xi-2$. In order to determine the amplitude of the $i_{v C, 2 \xi-2}$ harmonic, several dominant harmonic pairs of switching functions $s_{i_{-j}}$ and currents $i_{0, i_{-j}}$ were selected on the basis of Figure 8 and Table 5 .

Table 5. Conditions for harmonic components in dc-link of two parallel interleaved inverters.

The phase angles of the selected harmonic current components $i_{v C, k \pm k_{s}}^{(\operatorname{comp})}$ are equal or close to $2 \theta_{C 1 \_1}$ $\pm \pi / 2 \mathrm{rad}$.

Table 6 shows the coefficients of the selected harmonic currents and switching functions, and the components of the harmonic coefficient $I_{v C, 2 \xi-2}$ determined on the basis of Equations (39), (40), (42a), (42b), (42c), (51b) and (51c). Harmonic coefficient $I_{v C, 2 \xi-2}\left(I_{v C, 2 \xi+2}^{(o s)}=I_{v C, 2 \xi-2}\right)$ is described by the equation

$$
\begin{gathered}
I_{\nu C, 2 \xi-2}=\frac{3}{2} \frac{M U_{d c}}{\pi Z_{0,2 \xi}}\left[J_{3}(\pi M)-J_{1}(\pi M)\right]+\frac{3}{2} \frac{U_{D C}}{\pi^{2}} J_{1}(\pi M) J_{3}(2 \pi M)\left(\frac{1}{Z_{0,4 \xi}}-\frac{1}{Z_{0,2 \xi}}\right)+ \\
-\frac{8 U_{d c}}{3 \pi^{2} \omega_{C} L}\left\{9 J_{0}\left(\frac{\pi M}{2}\right) J_{2}\left(\frac{\pi M}{2}\right)+J_{2}\left(\frac{\pi M}{2}\right) J_{4}\left(\frac{3 \pi M}{2}\right)+J_{0}\left(\frac{\pi M}{2}\right) J_{2}\left(\frac{3 \pi M}{2}\right)+J_{0}\left(\frac{3 \pi M}{2}\right) J_{2}\left(\frac{\pi M}{2}\right)\right\}
\end{gathered}
$$


Table 6. Coefficients of the $i_{v C, 2 \xi-2}^{(c o m p)}$ and $i_{v C, 2 \xi+2}^{(c o m p)}$ components for the same (ss) and opposite (os) sequences of $u_{g i, 1}^{\prime}$ voltages and wave carriers, respectively.

\begin{tabular}{|c|c|c|c|}
\hline$S_{k_{s}}$ & $I_{o, k}$ & Coefficients of $i_{v C, 2 \xi-2}^{(\operatorname{comp})}$ and $i_{v C, 2 \xi+2}^{(\operatorname{comp})}$ Components & $\begin{array}{c}\phi_{v C, 2 \xi-2}= \\
\phi_{v C, 2 \xi+2}\end{array}$ \\
\hline $\begin{array}{l}m_{s}=1, n_{s}=0(\mathrm{p}) \\
m_{s}=1, n_{s}=0(\mathrm{n})\end{array}$ & $\begin{array}{c}m=1, n=-2(\mathrm{n}) \\
m=1, n=2(\mathrm{p})\end{array}$ & $\begin{array}{c}m_{s}+m=2, n_{s}+n=-2(\mathrm{ss}) \\
m_{s}+m=2, n_{s}+n=2(\mathrm{os})\end{array}$ & \\
\hline$(2 / \pi) J_{0}(\pi M / 2)$ & $-2 U_{d c} J_{2}(\pi M / 2) /\left(\omega_{C} L \pi\right)$ & $-12 U_{d c} J_{2}(\pi M / 2) J_{0}(\pi M / 2) /\left(\omega_{C} L \pi^{2}\right)$ & $2 \theta_{C 1 \_1}-\pi / 2$ \\
\hline $\begin{array}{c}m_{s}=1, n_{s}=-2(\mathrm{n}) \\
m_{s}=1, n_{s}=2(\mathrm{p})\end{array}$ & $\begin{array}{l}m=1, n=0(\mathrm{p}) \\
m=1, n=0(\mathrm{n})\end{array}$ & $\begin{array}{l}m_{s}+m=2, n_{s}+n=-2(\mathrm{ss}) \\
m_{s}+m=2, n_{s}+n=-2(\mathrm{os})\end{array}$ & \\
\hline$-(2 / \pi) J_{2}(\pi M / 2)$ & $2 U_{d c} J_{0}(\pi M / 2) /\left(\omega_{C} L \pi\right)$ & $-12 U_{d c} J_{2}(\pi M / 2) J_{0}(\pi M / 2) /\left(\omega_{C} L \pi^{2}\right)$ & $2 \theta_{C 1 \_1}-\pi / 2$ \\
\hline $\begin{array}{l}m_{s}=0, n_{s}=1(\mathrm{p}) \\
m_{s}=0, n_{s}=1(\mathrm{p})\end{array}$ & $\begin{array}{c}m=2, n=-1(\mathrm{p}) \\
m=2, n=1(\mathrm{n})\end{array}$ & $\begin{array}{c}m-m_{s}=2, n-n_{s}=-2(\mathrm{ss}) \\
m+m_{s}=2, n+n_{s}=2(\mathrm{os})\end{array}$ & \\
\hline$M / 2$ & $-U_{d c} J_{1}(\pi M) /\left(\pi Z_{o, 2 \xi}\right)$ & $-3 M U_{d c} J_{1}(\pi M) /\left(2 \pi Z_{o, 2 \xi}\right)$ & $2 \theta_{C 1 \_1}-\pi / 2$ \\
\hline $\begin{array}{l}m_{s}=0, n_{s}=1(\mathrm{p}) \\
m_{s}=0, n_{s}=1(\mathrm{p})\end{array}$ & $\begin{array}{c}m=2, n=-3(\mathrm{n}) \\
m=2, n=3(\mathrm{p})\end{array}$ & $\begin{array}{c}m+m_{s}=2, n+n_{s}=-2(\mathrm{ss}) \\
m-m_{s}=2, n-n_{s}=2(\mathrm{os})\end{array}$ & \\
\hline$M / 2$ & $U_{d c} J_{3}(\pi M) /\left(\pi Z_{o, 2 \xi}\right)$ & $3 M U_{d c} J_{3}(\pi M) /\left(2 \pi Z_{o, 2 \xi}\right)$ & $2 \theta_{C 1 \_1}-\pi / 2$ \\
\hline $\begin{array}{l}m_{S}=1, n_{S}=0(\mathrm{p}) \\
m_{S}=1, n_{S}=0(\mathrm{n})\end{array}$ & $\begin{array}{c}m=3, n=-2(\mathrm{p}) \\
m=3, n=2(\mathrm{n})\end{array}$ & $\begin{array}{c}m-m_{s}=2, n-n_{s}=-2(\mathrm{ss}) \\
m-m_{s}=2, n-n_{s}=2(\mathrm{os})\end{array}$ & \\
\hline$(2 / \pi) J_{0}(\pi M / 2)$ & $2 U_{d c} J_{2}(3 \pi M / 2) /\left(9 \pi \omega_{C} L\right)$ & $4 U_{d c} J_{0}(\pi M / 2) J_{2}(3 \pi M / 2) /\left(3 \omega_{C} L \pi^{2}\right)$ & $2 \theta_{C 1 \_1}-\pi / 2$ \\
\hline $\begin{array}{c}m_{s}=3, n_{s}=-4(\mathrm{n}) \\
m_{s}=3, n_{s}=4(\mathrm{p})\end{array}$ & $\begin{array}{c}m=1, n=-2(\mathrm{n}) \\
m=1, n=2(\mathrm{p})\end{array}$ & $\begin{array}{c}m_{s}-m=2, n_{s}-n=-2(\mathrm{ss}) \\
m_{s}-m=2, n_{s}-n=2(\mathrm{os})\end{array}$ & \\
\hline \multirow{2}{*}{$-2 J_{4}(3 \pi M / 2) /(3 \pi)$} & \multirow{2}{*}{$-2 U_{d c} J_{2}(\pi M / 2) /\left(\pi \omega_{C} L\right)$} & $4 U_{d c} J_{2}(\pi M / 2) J_{4}(3 \pi M / 2) /\left(\omega_{C} L \pi^{2}\right)$ & $2 \theta_{C 1 \_1}+\pi / 2$ \\
\hline & & $-4 U_{d c} J_{2}(\pi M / 2) J_{4}(3 \pi M / 2) /\left(\omega_{C} L \pi^{2}\right)$ & $2 \theta_{C 1 \_1}-\pi / 2$ \\
\hline $\begin{array}{l}m_{S}=1, n_{s}=-(\mathrm{n}) \\
m_{S}=1, n_{s}=2(\mathrm{p})\end{array}$ & $\begin{array}{c}m=3, n=-4(\mathrm{n}) \\
m=3, n=4(\mathrm{p})\end{array}$ & $\begin{array}{c}m-m_{s}=2, n-n_{s}=-2(\mathrm{ss}) \\
m-m_{s}=2, n-n_{s}=2(\mathrm{os})\end{array}$ & \\
\hline$-(2 / \pi) J_{2}(\pi M / 2)$ & $-2 U_{d c} J_{4}(3 \pi M / 2) /\left(9 \pi \omega_{C} L\right)$ & $4 U_{d c} J_{2}(\pi M / 2) J_{4}(3 \pi M / 2) /\left(3 \omega_{C} L \pi^{2}\right)$ & $2 \theta_{C 1 \_1}-\pi / 2$ \\
\hline $\begin{array}{c}m_{s}=3, n_{s}=-2(\mathrm{p}) \\
m_{s}=3, n_{s}=2(\mathrm{n})\end{array}$ & $\begin{array}{l}m=1, n=0(\mathrm{p}) \\
m=1, n=0(\mathrm{n})\end{array}$ & $\begin{array}{c}m_{s}-m=2, n_{s}-n=-2(\mathrm{ss}) \\
m_{s}-m=2, n_{s}-n=2(\mathrm{os})\end{array}$ & \\
\hline
\end{tabular}


Table 6. Cont

\begin{tabular}{|c|c|c|c|}
\hline$s_{k_{s}}$ & $I_{o, k}$ & Coefficients of $i_{v C, 2 \xi-2}^{(\operatorname{comp})}$ and $i_{v C, 2 \xi+2}^{(\operatorname{comp})}$ Components & $\begin{array}{c}\phi_{v C, 2 \xi-2}= \\
\phi_{v C, 2 \xi+2}\end{array}$ \\
\hline $2 J_{2}(3 \pi M / 2) /(3 \pi)$ & $2 U_{d c} J_{0}(\pi M / 2) /\left(\pi \omega_{C} L\right)$ & $4 U_{d c} J_{0}(\pi M / 2) J_{2}(3 \pi M / 2) /\left(\omega_{C} L \pi^{2}\right)$ & $2 \theta_{C 1 \_1}+\pi / 2$ \\
\hline $\begin{array}{l}m_{s}=3, n_{s}=0(0) \\
m_{s}=3, n_{s}=0(0)\end{array}$ & $\begin{array}{l}m=1, n=2(0) \\
m=1, n=-2(0)\end{array}$ & $\begin{array}{c}m_{s}-m=2, n_{s}-n=-2 ;(\mathrm{ss}) \\
m_{s}-m=2, n_{s}-n=2 ;(\mathrm{os})\end{array}$ & \\
\hline$-2 J_{0}(3 \pi M / 2) /(3 \pi)$ & $-2 U_{d c} J_{2}(\pi M / 2) /\left(\pi \omega_{C} L\right)$ & $4 U_{d c} J_{0}(3 \pi M / 2) J_{2}(\pi M / 2) /\left(\omega_{C} L \pi^{2}\right)$ & $2 \theta_{C 1 \_1}+\pi / 2$ \\
\hline $\begin{array}{c}m_{s}=1, n_{s}=2(0) \\
m_{s}=1, n_{s}=-2(0)\end{array}$ & $\begin{array}{l}m=3, n=0(0) \\
m=3, n=0(0)\end{array}$ & $\begin{array}{c}m-m_{s}=2, n-n_{s}=-2(\mathrm{ss}) \\
m-m_{s}=2, n-n_{s}=2(\mathrm{os})\end{array}$ & \\
\hline$-(2 / \pi) J_{2}(\pi M / 2)$ & $-2 U_{d c} J_{0}(3 \pi M / 2) /\left(9 \pi \omega_{C} L\right)$ & $4 U_{d c} J_{0}(3 \pi M / 2) J_{2}(\pi M / 2) /\left(3 \omega_{C} L \pi^{2}\right)$ & $2 \theta_{C 1 \_1}-\pi / 2$ \\
\hline $\begin{array}{l}m_{S}=2, n_{S}=-1(\mathrm{p}) \\
m_{S}=2, n_{S}=1(\mathrm{n})\end{array}$ & $\begin{array}{c}m=4, n=-3(\mathrm{p}) \\
m=4, n=3(\mathrm{n})\end{array}$ & $\begin{array}{l}m-m_{s}=2, n-n_{s}=-2(\mathrm{ss}) \\
m-m_{s}=2, n-n_{s}=-2(\mathrm{os})\end{array}$ & \\
\hline$-J_{1}(\pi M) / \pi$ & $-U_{d c} J_{3}(2 \pi M) /\left(2 \pi Z_{o, 4 \xi}\right)$ & $3 U_{d c} J_{1}(\pi M) J_{3}(2 \pi M) /\left(2 \pi^{2} Z_{o, 4 \xi}\right)$ & $2 \theta_{C 1 \_1}-\pi / 2$ \\
\hline $\begin{array}{c}m_{s}=4, n_{s}=-3(\mathrm{p}) \\
m_{s}=4, n_{s}=3(\mathrm{n})\end{array}$ & $\begin{array}{l}m=2, n=-1(\mathrm{p}) \\
m=2, n=1(\mathrm{n})\end{array}$ & $\begin{array}{c}m_{s}-m=2, n_{s}-n=-2(\mathrm{ss}) \\
m_{s}-m=2, n_{s}-n=2(\mathrm{os})\end{array}$ & \\
\hline$-J_{3}(2 \pi M) /(2 \pi)$ & $-U_{d c} J_{1}(\pi M) /\left(\pi Z_{0,2 \xi}\right)$ & $3 U_{d c} J_{1}(\pi M) J_{3}(2 \pi M) /\left(2 \pi^{2} Z_{o, 2 \xi}\right)$ & $2 \theta_{C 1 \_1}+\pi / 2$ \\
\hline
\end{tabular}


If Equation (45) is used and both $Z_{o, 2 \xi}$ and $Z_{o, 4 \xi}$ are shown in simplified forms $2 \omega_{C} L$ and $4 \omega_{C} L$, we obtain

$$
I_{v C, 2 \xi-2}=-\frac{U_{d c}}{\omega_{C} L} H_{2 \xi-2}
$$

where

$$
H_{2 \xi-2}=\frac{8}{3 \pi^{2}}\left\{J_{2}\left(\frac{\pi M}{2}\right)\left[9 J_{0}\left(\frac{\pi M}{2}\right)+J_{4}\left(\frac{3 \pi M}{2}\right)+J_{0}\left(\frac{3 \pi M}{2}\right)\right]+J_{0}\left(\frac{\pi M}{2}\right) J_{2}\left(\frac{3 \pi M}{2}\right)+\frac{9}{32} \pi M\left[J_{1}(\pi M)-J_{3}(\pi M)\right]+\frac{9}{64} J_{1}(\pi M) J_{3}(2 \pi M)\right\}
$$

The other harmonic coefficients may be determined likewise.

$$
I_{v C, l}=\frac{U_{d c}}{\omega_{C} L} H_{l}
$$

On the basis of simulation tests for $M=0.05,0.225,0.45,0.675$, and 0.9 , the $I_{v C, k}$ harmonic spectra for $k \in(1-20 \xi)$ were determined and the harmonic coefficients of these orders were selected, for which the maximum rms values were not lower than $15 \%$ of the maximum rms value of the $i_{d c c}$ current.

Table A1 (see Appendix A) contains the values of the $H_{l}(M)$ coefficients (depending on the depth of modulation $M$ ) occurring in the above equations.

The rms value of current component $i_{d c C}$ can be written as

$$
I_{d C,, r m s} \approx 1.1 \frac{\sqrt{2}}{2} \sqrt{\begin{array}{c}
\left(\hat{I}_{v C, 2 \xi-2}\right)^{2}+\left(\hat{I}_{v C, 2 \xi+4}\right)^{2}+\left(\hat{I}_{v C, 4 \xi-4}\right)^{2}+\left(\hat{I}_{v C, 4 \xi+2}\right)^{2}+2\left(\hat{I}_{v C, 6 \xi-6}\right)^{2}+\left(\hat{I}_{v C, 6 \xi}\right)^{2}+ \\
+\left(\hat{I}_{v C, 8 \xi-2}\right)^{2}+\left(\hat{I}_{v C, 8 \xi+4}\right)^{2}+\left(\hat{I}_{v C, 8 \xi-10}\right)^{2}+\left(\hat{I}_{v C, 10 \xi+2}\right)^{2}+\left(\hat{I}_{v C, 12 \xi}\right)^{2}+\left(\hat{I}_{v C, 18 \xi}\right)^{2}+\left(\hat{I}_{v C, 14 \xi+4}\right)^{2}
\end{array}}
$$

or in simplified form,

$$
I_{d c C, r m s} \approx 0.78 \frac{U_{d c}}{\omega_{C} L} G(M)
$$

where

$$
G(M)=\sqrt{\begin{array}{c}
\left(H_{v C, 2 \xi-2}\right)^{2}+\left(H_{v C, 2 \xi+4}\right)^{2}+\left(H_{v C, 4 \xi-4}\right)^{2}+\left(H_{v C, 4 \xi+2}\right)^{2}+2\left(H_{v C, 6 \xi-6}\right)^{2}+\left(H_{v C, 6 \xi}\right)^{2}+ \\
+\left(H_{v C, 8 \xi-2}\right)^{2}+\left(H_{v C, 8 \xi+4}\right)^{2}+\left(H_{v C, 8 \xi-10}\right)^{2}+\left(H_{v C, 10 \xi+2}\right)^{2}+\left(H_{v C, 12 \xi}\right)^{2}+\left(H_{v C, 18 \xi}\right)^{2}+\left(H_{v C, 14 \xi+4}\right)^{2}
\end{array}}
$$

The factor with a value of 1.1 in Equations (58) and (59), determined from simulation tests, takes into account a limited number of harmonics in Equations (58) and (60).

\section{Simulation Tests of the Power Supply System}

Simulation data was performed in the Turbo Pascal package. Simulation tests of the power supply system were performed with the parameters given in Table 7 . The value of the $L$ inductance ( $L \approx L_{-} \approx \approx L_{-} 2$ ) should be small enough to provide the required slew rate of the PAPF output current. On the other hand, the minimum value of $L$ is a function of parameters such as $f_{C}, U_{d c}$, and $\tau_{w}$, and the gain of the output current regulator of PAPF inverters [22]. The capacitance value $C$ (twice the capacitance value of the capacitors connected to dc-link) must ensure that the condition of Equation (17) is met. In [1], for the three-phase active rectifier system, and in [2], for a system with a three-parallel power converter, the value of the damping resistor $R_{d}=1 /\left(6 \pi f_{\text {res }} C\right)$ was adopted, from which the resonant pole damping factor $\zeta_{p}=1 / 6$ and $R_{d}=180 \mathrm{~m} \Omega$ can be concluded. 
Table 7. Basic grid and converter parameters.

\begin{tabular}{ll}
\hline Parameter & Value \\
\hline$f_{g}, L_{g}^{\prime}$. (grid dispersion inductance) & $50 \mathrm{~Hz}, 3 \mu \mathrm{H}$ \\
$T r 1, v_{z}$ & Dy5 $(400 \mathrm{~V} / 400 \mathrm{~V}), \sqrt{ } 3$ \\
$T r 2$ & Dd0y5 $(400 \mathrm{~V} / 400 \mathrm{~V} / 400 \mathrm{~V})$ \\
$L_{l q 2}^{\prime}(T h 1-T h 12$ rectifier side dispersion inductance of $\operatorname{Tr} 2)$ & $80 \mu \mathrm{H}$ \\
$L_{a}^{\prime}, R_{a}^{\prime}, I_{a}^{\prime}, U_{a}^{\prime}$ & $300 \mu \mathrm{H}, 3 \mathrm{~m} \Omega, 1035 \mathrm{~A}, 644 \mathrm{~V}$ \\
Firing angle of $S C R$ converter $\alpha$ & $50^{\circ}$ \\
$U_{d c}, C_{d c}$ & $720 \mathrm{~V}, 2.7 \mathrm{mF}$ \\
$L_{-}, L_{-2}, L$ & $76 \mu \mathrm{H}, 84 \mu \mathrm{H}, 80 \mu \mathrm{H}$ \\
$C_{,} R_{d}, L_{l q}(A P F$ side dispersion inductance of $\operatorname{Tr} 1)$ & $0 \mu \mathrm{F}, 180 \mathrm{~m} \Omega, 20 \mu \mathrm{H}$ \\
\hline Control system: & \\
\hline$f_{C}$ (carrier frequency) & $10 \mathrm{kHz}, 15 \mathrm{kHz}$ \\
$f_{\text {samp }}$ (carrier frequency and sampling frequency) & $20 \mathrm{kHz}, 30 \mathrm{kHz}$ \\
\hline
\end{tabular}

Figure 9a,b shows voltage and current waveforms obtained from simulation tests for the compensation system shown in Figure 1a with the control system described in [22]. The load current, the line current, the mains voltage source and the rectified voltage $u_{a}$ are brought to transformer $\operatorname{Tr} 1$ primary side terminals. The simulation (Figure 9a,b) was performed for the same sequence $u_{g 1,1}^{\prime}, u_{g 2,1}^{\prime}$ and $u_{g 3,1}^{\prime}$ voltages and wave carriers with programmed compensation 11, 13, 23 and 25 harmonics. The amplitudes and initial phases of individual harmonic voltages and currents, which are shown by the waveforms in Figure 9a,b, are given in Table 8.

The results shown in Table 8 allow for the verification of Equations (8), (11), (13) and (29b) that determine amplitudes $\hat{I}_{\mathcal{C}, k}$ and $\hat{I}_{\mathcal{L}, k, k}$, and rms values $I_{\mathcal{C} G L, r m s}$ and $I_{\mathcal{C} C, r m s}$, respectively. Tests will be used to calculate the value of $I_{\mathcal{C}, r m s}$ input data for simulation $\left(L_{-} 1=L_{-} 2=L, C, L_{l q}, L_{g}, R_{d}, f_{C}, f_{g}, v_{z}\right)$. Amplitude values $\hat{U}_{g 1, k}$ and $\hat{I}_{L 1, k}$ and initial phases $\psi_{g 1, k}$ and $\phi_{L 1, k}$ should be measured under real conditions. For $\hat{U}_{g 1, k}^{\prime}=326.6 \mathrm{~V}$ and $U_{d c}=720 \mathrm{~V}$ we obtain $M \approx 0.9$. After placing data into Equation (29b) we obtain $I_{c C, r m s}=14.8 \mathrm{~A}$ (calculated values based on the harmonic amplitudes of orders 597, 599, 601 and 603, are shown in Table 8 as 13.3 A).

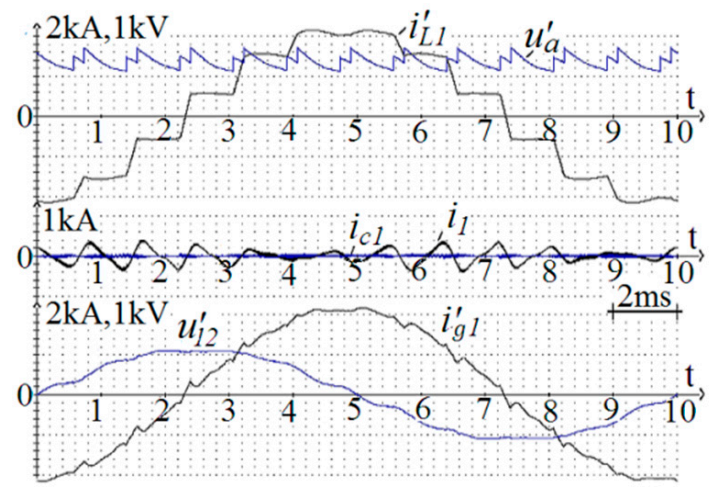

(a)

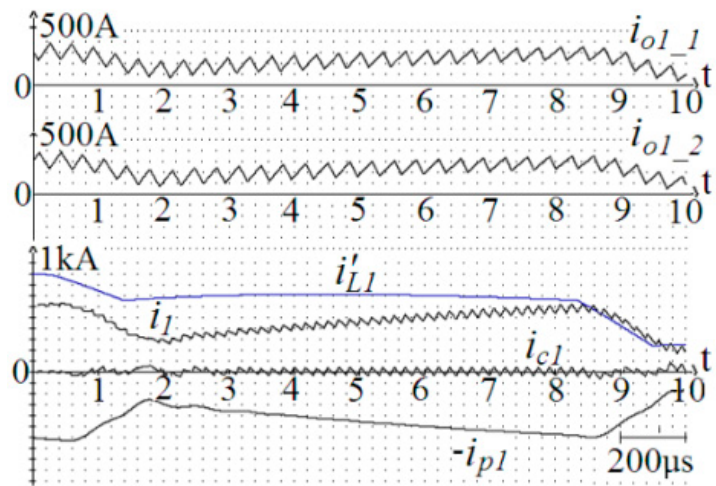

(b)

Figure 9. (a) Simulation of waveforms of currents: $i_{L 1}^{\prime}, i_{1}, i_{g 1}^{\prime}$, and $i_{c 1}$ and voltages $u_{a}^{\prime}$ and $u_{12}^{\prime}$; and (b) simulation of the inverter output currents $i_{01-1}$ and $i_{01-2}$ and their sum $i_{1}$, output current of the PAPF $i_{p 1}$, load current $i_{L 1}$, and filter capacitor current $i_{c 1}$. 
Table 8. Amplitudes and initial phases of individual harmonics in the system with full load.

\begin{tabular}{|c|c|c|c|c|c|c|c|c|c|c|c|c|}
\hline$k, 1$ & $\hat{U}_{g 1, k}^{\prime}$ & $\psi_{g 1, k}^{\prime}$ & $\hat{I}_{g 1, k}^{\prime}$ & $\hat{I}_{L 1, k}^{\prime}$ & $\varphi_{L 1, k}^{\prime}$ & $\hat{I}_{1, k}$ & $\varphi_{1, k}^{\prime}$ & $\hat{I}_{01 \_1, k}$ & $\hat{I}_{c 1, k}$ & $\hat{I}_{c p, k}$ & $\hat{I}_{v, l}$ & $\hat{I}_{d c, l}$ \\
\hline & V & $\mathrm{rad}$ & A & A & $\mathrm{rad}$ & A & rad & A & A & A & A & A \\
\hline 1 & 326.6 & 0.000 & 2162.3 & 2156.6 & -0.926 & 5.9 & 1.398 & 2.8 & 5.1 & 0.0 & 0.2 & 0.2 \\
\hline 2 & 0.0 & 0.000 & 0.1 & 0.2 & 1.710 & 0.3 & 1.173 & 0.2 & 0.0 & 0.0 & 0.2 & 0.2 \\
\hline 3 & 0.0 & 0.000 & 1.0 & 1.1 & 2.154 & 1.2 & 2.769 & 0.6 & 0.2 & 0.3 & 1.3 & 1.2 \\
\hline 4 & 0.0 & 0.000 & 0.3 & 0.1 & 1.524 & 0.5 & 2.193 & 0.2 & 0.0 & 0.0 & 0.4 & 0.4 \\
\hline 5 & 0.0 & 0.000 & 17.9 & 18.7 & 2.716 & 2.7 & -2.90 & 1.4 & 0.0 & 0.0 & 0.3 & 0.4 \\
\hline 6 & 0.0 & 0.000 & 0.4 & 0.1 & 0.000 & 0.5 & 2.727 & 0.2 & 0.0 & 0.0 & 4.4 & 4.4 \\
\hline 7 & 0.0 & 0.000 & 5.8 & 10.5 & -0.900 & 4.6 & -0.391 & 2.4 & 0.0 & 0.0 & 0.3 & 0.4 \\
\hline 8 & 0.0 & 0.000 & 0.7 & 0.1 & 0.000 & 0.6 & 2.510 & 0.4 & 0.0 & 0.1 & 0.3 & 0.4 \\
\hline 9 & 0.0 & 0.000 & 2.0 & 1.5 & -0.398 & 2.4 & 1.761 & 1.1 & 1.0 & 1.5 & 2.8 & 2.5 \\
\hline 10 & 0.0 & 0.000 & 0.8 & 0.2 & -1.507 & 0.7 & 1.981 & 0.5 & 0.0 & 0.1 & 0.2 & 0.2 \\
\hline 11 & 12.0 & -2.967 & 53.5 & 206.7 & -0.719 & 169.1 & -1.462 & 84.6 & 4.0 & 0.1 & 0.5 & 0.5 \\
\hline 12 & 0.0 & 0.000 & 0.6 & 0.1 & 0.000 & 0.1 & -0.275 & 0.2 & 0.1 & 0.2 & 163.5 & 163.6 \\
\hline 13 & 8.0 & -0.349 & 5.8 & 129.6 & 0.440 & 124.3 & 0.909 & 62.4 & 3.4 & 0.2 & 0.6 & 0.7 \\
\hline 14 & 0.0 & 0.000 & 0.4 & 0.1 & -2.398 & 0.7 & 1.858 & 0.3 & 0.1 & 0.1 & 0.6 & 0.7 \\
\hline 15 & 0.0 & 0.000 & 1.3 & 1.4 & -2.762 & 2.2 & -0.757 & 1.0 & 1.8 & 2.8 & 3.8 & 2.5 \\
\hline 16 & 0.0 & 0.000 & 0.3 & 0.1 & 2.915 & 0.2 & 1.778 & 0.2 & 0.1 & 0.1 & 0.7 & 0.8 \\
\hline 17 & 0.0 & 0.000 & 14.5 & 10.4 & -1.839 & 4.0 & 0.376 & 2.0 & 0.1 & 0.0 & 0.9 & 0.9 \\
\hline 18 & 0.0 & 0.000 & 1.3 & 0.0 & 0.000 & 1.2 & -2.740 & 0.6 & 0.1 & 0.1 & 4.5 & 4.6 \\
\hline 19 & 0.0 & 0.000 & 12.1 & 8.5 & -0.423 & 3.7 & 3.070 & 2.0 & 0.3 & 0.3 & 1.1 & 0.9 \\
\hline 20 & 0.0 & 0.000 & 0.9 & 0.1 & 0.000 & 0.7 & -2.559 & 0.3 & 0.4 & 0.5 & 1.1 & 0.6 \\
\hline 21 & 0.0 & 0.000 & 1.8 & 1.3 & 1.039 & 1.9 & -1.155 & 0.9 & 1.2 & 1.9 & 2.5 & 1.6 \\
\hline 22 & 0.0 & 0.000 & 0.5 & 0.2 & -0.092 & 1.4 & -1.177 & 0.5 & 0.3 & 0.5 & 1.0 & 0.9 \\
\hline 23 & 0.0 & 0.000 & 10.0 & 70.5 & 0.663 & 56.6 & 0.159 & 28.2 & 3.1 & 0.2 & 2.0 & 2.2 \\
\hline 24 & 0.0 & 0.000 & 1.3 & 0.1 & 0.000 & 1.2 & -1.442 & 0.7 & 0.3 & 0.5 & 33.1 & 33.0 \\
\hline 25 & 0.0 & 0.000 & 14.7 & 57.9 & 1.957 & 40.6 & 2.568 & 20.3 & 3.3 & 0.7 & 1.9 & 2.4 \\
\hline 298 & 0.0 & 0.000 & 0.0 & 0.0 & 0.000 & 0.1 & 1.171 & 13.1 & 0.1 & 0.0 & 0.1 & 0.1 \\
\hline 299 & 0.0 & 0.000 & 0.4 & 0.3 & -2.041 & 0.1 & 0.000 & 1.2 & 0.0 & 0.0 & 0.0 & 0.0 \\
\hline 300 & 0.0 & 0.000 & 0.0 & 0.0 & 0.000 & 0.0 & 0.000 & 32.9 & 0.0 & 0.0 & 0.2 & 0.2 \\
\hline 301 & 0.0 & 0.000 & 0.5 & 0.4 & -0.816 & 0.1 & 0.000 & 1.2 & 0.0 & 0.1 & 0.2 & 0.0 \\
\hline 302 & 0.0 & 0.000 & 0.0 & 0.0 & 0.000 & 0.1 & 0.000 & 11.5 & 0.1 & 0.1 & 0.2 & 0.1 \\
\hline 550 & 0.0 & 0.000 & 0.0 & 0.0 & 0.000 & 0.2 & 0.170 & 0.2 & 0.2 & 0.0 & 6.3 & 6.2 \\
\hline 562 & 0.0 & 0.000 & 0.0 & 0.0 & 0.000 & 0.6 & -0.330 & 0.3 & 0.6 & 0.2 & 9.4 & 9.6 \\
\hline 568 & 0.0 & 0.000 & 0.0 & 0.0 & 0.000 & 0.5 & -1.247 & 0.3 & 0.5 & 0.3 & 7.4 & 7.1 \\
\hline 574 & 0.0 & 0.000 & 0.0 & 0.0 & 0.000 & 0.3 & 3.123 & 0.2 & 0.3 & 0.3 & 6.9 & 6.9 \\
\hline 580 & 0.0 & 0.000 & 0.0 & 0.0 & 0.000 & 0.2 & -0.967 & 0.1 & 0.2 & 0.2 & 6.4 & 6.5 \\
\hline 589 & 0.0 & 0.000 & 0.0 & 0.0 & 0.000 & 2.6 & 0.977 & 1.3 & 2.6 & 4.2 & 4.7 & 0.5 \\
\hline 592 & 0.0 & 0.000 & 0.0 & 0.0 & 0.000 & 0.1 & -0.017 & 0.1 & 0.2 & 0.4 & 17.0 & 16.8 \\
\hline 595 & 0.0 & 0.000 & 0.1 & 0.1 & 0.000 & 2.2 & -0.410 & 1.1 & 2.2 & 3.5 & 2.8 & 0.6 \\
\hline 597 & 0.0 & 0.000 & 0.4 & 0.0 & 0.000 & 7.5 & -2.483 & 3.8 & 7.8 & 0.4 & 1.2 & 1.5 \\
\hline 598 & 0.0 & 0.000 & 0.0 & 0.0 & 0.000 & 1.2 & 1.269 & 0.6 & 1.3 & 0.5 & 30.3 & 30.8 \\
\hline 599 & 0.0 & 0.000 & 0.5 & 0.0 & 0.000 & 9.8 & 1.862 & 4.9 & 10.1 & 0.6 & 2.7 & 2.1 \\
\hline 600 & 0.0 & 0.000 & 0.1 & 0.0 & 0.000 & 0.2 & 0.407 & 0.1 & 0.2 & 0.9 & 2.2 & 1.3 \\
\hline 601 & 0.0 & 0.000 & 0.1 & 0.1 & 0.000 & 11.4 & 2.807 & 5.7 & 11.4 & 17.9 & 18.5 & 1.1 \\
\hline 602 & 0.0 & 0.000 & 0.1 & 0.0 & 0.000 & 1.4 & 0.058 & 0.7 & 1.4 & 1.2 & 0.9 & 0.4 \\
\hline 603 & 0.0 & 0.000 & 0.3 & 0.0 & 0.000 & 7.6 & 0.767 & 3.8 & 7.9 & 0.5 & 0.6 & 0.9 \\
\hline 604 & 0.0 & 0.000 & 0.0 & 0.0 & 0.000 & 0.5 & -1.395 & 0.2 & 0.5 & 0.2 & 12.1 & 12.3 \\
\hline 609 & 0.0 & 0.000 & 0.1 & 0.0 & 0.000 & 2.3 & 1.266 & 1.2 & 2.4 & 0.1 & 1.7 & 1.7 \\
\hline 610 & 0.0 & 0.000 & 0.0 & 0.0 & 0.000 & 0.3 & -2.500 & 0.2 & 0.3 & 0.0 & 25.1 & 25.1 \\
\hline 613 & 0.0 & 0.000 & 0.1 & 0.1 & 0.000 & 3.0 & -0.720 & 1.5 & 3.0 & 4.5 & 3.9 & 0.7 \\
\hline 616 & 0.0 & 0.000 & 0.0 & 0.0 & 0.000 & 0.2 & -2.064 & 0.1 & 0.2 & 0.3 & 22.3 & 22.3 \\
\hline 622 & 0.0 & 0.000 & 0.0 & 0.0 & 0.000 & 0.2 & 1.651 & 0.1 & 0.3 & 0.2 & 21.5 & 21.4 \\
\hline 628 & 0.0 & 0.000 & 0.0 & 0.0 & 0.000 & 0.2 & -0.442 & 0.1 & 0.2 & 0.0 & 5.3 & 5.3 \\
\hline 634 & 0.0 & 0.000 & 0.0 & 0.0 & 0.000 & 0.3 & 2.551 & 0.2 & 0.3 & 0.1 & 8.6 & 8.6 \\
\hline 640 & 0.0 & 0.000 & 0.0 & 0.0 & 0.000 & 0.3 & 2.546 & 0.2 & 0.3 & 0.1 & 6.8 & 7.0 \\
\hline 646 & 0.0 & 0.000 & 0.0 & 0.0 & 0.000 & 0.1 & 0.000 & 0.1 & 0.1 & 0.1 & 7.2 & 7.3 \\
\hline 652 & 0.0 & 0.000 & 0.0 & 0.0 & 0.000 & 0.1 & 0.519 & 0.1 & 0.1 & 0.1 & 5.8 & 5.8 \\
\hline
\end{tabular}




\subsection{Rms of Capacitor C Current}

Table 9 contains further results of simulation research $\varphi_{g 1,1}$ is the initial phase of the fundamental harmonic current $i g_{1}$ ). Table 10 combines the results of simulations for compensated and uncompensated $(k=1)$ harmonics of the load current. For compensated harmonics, the value $\hat{I}_{c L, k}$ was determined based on Equation (11) and $\lambda_{k}>0$, but for uncompensated harmonics the same equation was used with $\lambda_{k}=0$. On the basis of Equation (13) and the data given in Table 8, $I_{c G L 1, k(r m s)}$ was calculated for $k=1,11,13,23$, and 25 (Table 10). Based on these harmonics, $I_{c G L, r m s}=5.9$ A was obtained.

Table 9. Further results of simulation research for the system with full load.

\begin{tabular}{|c|c|c|c|c|c|c|c|}
\hline$I_{g 1(r m s)}^{\prime}$ & $I_{1(\mathrm{rms})}$ & $\varphi_{g 1,1}^{\prime}$ & $I_{c 1(r m s)}$ & $I_{d c(r m s)}$ & $I_{L 1(r m s)}^{\prime}$ & $U_{12(r m s)}^{\prime}$ & $U_{g(r m s)}^{\prime}$ \\
\hline $1531 \mathrm{~A}$ & $155.1 \mathrm{~A}$ & $-0.93 \mathrm{rad}$ & $18.4 \mathrm{~A}$ & $138 \mathrm{~A}$ & $1536 \mathrm{~A}$ & $397 \mathrm{~V}$ & $231 \mathrm{~V}$ \\
\hline \multicolumn{2}{|c|}{$T H D_{i L 1}=12.3 \%$} & \multicolumn{2}{|c|}{$T H D_{i g 1}=4.36 \%$} & \multicolumn{2}{|c|}{$T H D_{u 12}=4.62 \%$} & \multicolumn{2}{|c|}{$T H D_{u g 1}=4.42 \%$} \\
\hline
\end{tabular}

Table 10. Results of simulation research and calculations for the system with full load.

\begin{tabular}{|c|c|c|c|c|c|c|c|c|c|}
\hline \multicolumn{5}{|c|}{ Results of Simulation Research } & \multicolumn{5}{|c|}{ Results of Calculations } \\
\hline$k$ & $\hat{U}_{g 1, k}^{\prime}$ & $\Psi_{g 1, k}^{\prime}$ & $\hat{I}_{L 1, k}^{\prime}$ & $\varphi_{L 1, k}^{\prime}$ & $I_{c 1, k(r m s)}$ & $\lambda_{k}$ & $\hat{I}_{c G 1, k}$ & $\hat{I}_{c L 1, k}$ & $I_{c G L 1, k(r m s)}$ \\
\hline- & $\mathrm{V}$ & $\mathrm{rad}$ & A & $\mathrm{rad}$ & $\mathrm{A}$ & - & $\mathrm{A}$ & $\mathrm{A}$ & $\mathrm{A}$ \\
\hline 1 & 327 & 0 & 2156.6 & -0.93 & 3.6 & 0 & 5.13 & 0.032 & 3.64 \\
\hline 11 & 12 & -2.97 & 206.7 & -0.72 & 2.8 & 0.82 & 2.1 & 1.98 & 2.72 \\
\hline 13 & 8 & -0.35 & 129.6 & 0.44 & 2.4 & 0.96 & 1.66 & 2.1 & 2.46 \\
\hline 23 & 0 & & 70.5 & 0.66 & 2.2 & 0.8 & 0 & 3.0 & 2.12 \\
\hline 25 & 0 & & 57.9 & 2.00 & 2.33 & 0.7 & 0 & 2.52 & 1.78 \\
\hline
\end{tabular}

On the basis of Equations (13), (29b), and (30), a current of $I_{c, r m s}=15.9$ A was determined (the value given in Table 9 calculated in the numerical model for the course of the instantaneous capacitor current is $18.4 \mathrm{~A}$ ). The value calculated is $15 \%$ lower than the value determined numerically for the instantaneous course of the capacitor current. To achieve good accuracy of Equation (13), which takes into account that the number of harmonics in the voltage and load current is limited to just 40 , a good damping of the LCL-filter for a PAPF is required, eliminating the phenomenon of resonance.

\subsection{Rms Value of Ripple Current in Dc-Link Capacitors}

Based on Equation (57) and Table A1, harmonic coefficients $I_{v C, 2 \xi-2}, I_{v C, 2 \xi+4}, I_{v C, 4 \xi-4}, I_{v C, 4 \xi+2}$, $I_{v C, 6 \xi-6}, I_{v C, 6 \xi}$, and $I_{v C, 6 \xi+6}$ can be determined for different coefficients of modulation depth $\mathrm{M}$. The values of the amplitudes calculated in this way and the amplitudes determined in the numerical model are presented in Table 11. The main source of error in the calculations given in Table 11 is that the $H_{l}(M)$ harmonic factors describing these harmonics were determined on the basis of a limited number of harmonic pairs of the switching function and the output currents of the inverter branches. The second source of error is the lack of consideration of harmonic components forced by the active current taken from the grid and related to thermal losses in the inverters.

Table 12 contains the results of simulation tests carried out for the control system of the PAPF output current with asymmetrical and symmetrical regular-sampled PWM.

The $I_{d c C, r m s}$ value determined from Equation (59) for $f_{C}=15 \mathrm{kHz}$ and $M=0.9$ is $35.2 \mathrm{~A}$ (the value given in Table 12 calculated in the numerical model for the course of the instantaneous capacitor $C_{d c}$ current is $36.2 \mathrm{~A}$ ). 
Table 11. Results of simulation research and calculations in the system without load.

\begin{tabular}{|c|c|c|c|c|c|c|c|c|c|c|c|c|c|c|}
\hline \multicolumn{15}{|c|}{ Results of Simulation Research/Results of Calculations } \\
\hline$f_{C}[\mathrm{kHz}]$ & 15 & 10 & 15 & 10 & 15 & 10 & 15 & 10 & 15 & 10 & 15 & 10 & 15 & 10 \\
\hline$\hat{I}_{v C, k}$ & \multicolumn{2}{|c|}{$\hat{I}_{\nu C, 2 \xi-2}[A]$} & \multicolumn{2}{|c|}{$\hat{I}_{v C, 2 \xi+4}[A]$} & \multicolumn{2}{|c|}{$\hat{I}_{v C, 4 \xi-4}[A]$} & \multicolumn{2}{|c|}{$\hat{I}_{\nu C, 4 \xi+2}[A]$} & \multicolumn{2}{|c|}{$\hat{I}_{\nu C, 6 \xi-6}[A]$} & \multicolumn{2}{|c|}{$\hat{I}_{v C, 6 \xi}[A]$} & \multicolumn{2}{|c|}{$\hat{I}_{v C, 6 \xi+6}[A]$} \\
\hline $\mathrm{M}=$ & $34.9 /$ & $50.1 /$ & $19.0 /$ & 29.1/ & 17.7/ & $26.5 /$ & $2.8 /$ & $2.8 /$ & $10.0 /$ & $14.3 /$ & $2.6 /$ & $3.7 /$ & $9.8 /$ & $13.8 /$ \\
\hline 0.9 & 34.2 & 51.3 & 18.4 & 27.7 & 18.5 & 27.8 & 1.2 & 1.9 & 7.9 & 11.9 & 1.8 & 2.7 & 7.9 & 11.9 \\
\hline$M=$ & $25.7 /$ & $39.8 /$ & $2.1 /$ & $3.6 /$ & $7.7 /$ & $12.5 /$ & $30.0 /$ & $45.4 /$ & $3.5 /$ & $5.4 /$ & $14.7 /$ & 22.1/ & $3.5 /$ & $5.1 /$ \\
\hline 0.45 & 26.7 & 40.1 & 2.1 & 3.2 & 8.0 & 12.0 & 29.0 & 43.5 & 3.3 & 4.9 & 13.7 & 20.5 & 3.3 & 4.9 \\
\hline$M=$ & $1.06 /$ & $1.4 /$ & $0.07 /$ & $0.1 /$ & $0.07 /$ & $0.0 /$ & $1.35 /$ & $1.7 /$ & $0.2 /$ & $0.5 /$ & $45.5 /$ & $67.7 /$ & $0.2 /$ & $0.5 /$ \\
\hline 0.05 & 0.5 & 0.7 & 0 & 0 & 0 & 0 & 0.9 & 1.3 & 0 & 0 & 43.7 & 65.6 & 0 & 0 \\
\hline
\end{tabular}

Table 12. The currents of LCL-filter and dc-link capacitors and common mode voltage in an PAPF with one and two inverters without load for a positive sequence of fundamental voltages $u_{g 1,1}^{\prime}, u_{g 2,1}^{\prime}$ and $u_{g 3,1}^{\prime}$.

\begin{tabular}{|c|c|c|c|c|c|c|c|c|c|c|c|c|}
\hline \multirow{2}{*}{$f_{C}=15 \mathrm{kHz}$} & \multicolumn{12}{|c|}{ Results for Asymmetrical Regular Sampled PWM/Results for Symmetrical Regular Sampled PWM } \\
\hline & \multicolumn{2}{|c|}{ PAPF with One Three-Phase Voltage Source Inverter } & \multicolumn{10}{|c|}{ PAPF with Two Paralleled Three-Phase Voltage Source Inverters } \\
\hline Converter-Side Inductances & \multicolumn{2}{|c|}{$L / 2$} & \multicolumn{10}{|c|}{$L$} \\
\hline \multirow{3}{*}{$\begin{array}{c}\begin{array}{c}\text { Carrier Based PWM } \\
\text { Strategies }\end{array} \\
M\end{array}$} & \multirow{2}{*}{ Single PWM Carrier } & \multirow{2}{*}{$\begin{array}{l}\text { Three Interleaved } \\
\text { PWM Carriers [32] }\end{array}$} & \multirow{3}{*}{$\begin{array}{l}\text { Single PWM } \\
\text { Carrier }\end{array}$} & \multirow{2}{*}{\multicolumn{4}{|c|}{ Double Interleaved PWM Carriers [3] }} & \multicolumn{5}{|c|}{ Double Three Interleaved PWM Carriers [13] } \\
\hline & & & & & & & & \multicolumn{4}{|c|}{ Positive Sequence Carrier } & \multirow{2}{*}{$\begin{array}{c}\text { Negative Sequence Carrier } \\
0.9\end{array}$} \\
\hline & & 0.9 & & 0.9 & 0.675 & 0.45 & 0.05 & 0.9 & 0.675 & 0.45 & 0.05 & \\
\hline \multirow{2}{*}{$I_{c, r m s}[\mathrm{~A}]$} & $61.9 /$ & $67.37 /$ & $62.0 /$ & $17.0 /$ & $19.2 /$ & $18.0 /$ & $2.88 /$ & $17.1 /$ & $19.0 /$ & $17.8 /$ & $2.88 /$ & $17.0 /$ \\
\hline & 61.8 & 67.24 & 61.7 & 16.3 & 19.2 & 18.0 & 3.4 & 16.3 & 19.0 & 17.8 & 3.4 & 16.3 \\
\hline \multirow[b]{2}{*}{$I_{d c, r m s}[\mathrm{~A}]$} & 53.1/ & $56.12 /$ & $53.3 /$ & $31.3 /$ & $41.2 /$ & $64.9 /$ & $123 /$ & 36.21 & $40.43 /$ & $36.8 /$ & 37.71 & $36.4 /$ \\
\hline & 52.4 & 55.06 & 52.4 & 30.2 & 41.3 & 64.9 & 121 & 35.2 & 39.38 & 36.9 & 37.4 & 36.2 \\
\hline \multirow{2}{*}{$C M V_{r m s}[\mathrm{~V}]$} & $14.4 /$ & $6.32 /$ & $14.4 /$ & $4.4 /$ & $1.17 /$ & $0.60 /$ & $0.04 /$ & $4.33 /$ & $2.57 /$ & $2.5 /$ & $0.39 /$ & $4.3 /$ \\
\hline & 15.2 & 7.99 & 15.2 & 8.9 & 8.26 & 8.22 & 8.25 & 8.79 & 8.6 & 8.56 & 8.25 & 8.89 \\
\hline$N$ & 3 & 1 & 6 & \multicolumn{4}{|c|}{6} & \multicolumn{5}{|c|}{2} \\
\hline
\end{tabular}


6.3. The Currents of LCL-Ripple Filter and Dc-Link Capacitors in a PAPF with One and Two Voltage Source Inverters for Positive and Negative Sequence Carriers

Simulation tests were carried out with inductors $L_{-1}$ and $L_{-} 2$ with identical inductance values equal to $L$ in a system with two inverters and inductors with inductance values equal to half of $L$ in a system with one inverter. This choice of inductance values means the same resonance frequency of the LCL filter in systems with two and one inverters.

Simulation research shows that the harmonic spectrum of currents $i_{d c C}$ changes with the change of carrier sequence, but the rms current value of the $C_{d c}$ capacitor does not change (Table 12). This is due to the equality of the relevant harmonic coefficients: $I_{v C, 2 \xi+2}^{(o s)}=I_{v C, 2 \xi-2}, I_{v C, 4 \xi+4}^{(o s)}=I_{v C, 4 \xi-4}$, $I_{v C, 2 \xi-4}^{(o s)}=I_{v C, 2 \xi+4}, I_{v C, 4 \xi-2}^{(o s)}=I_{v C, 4 \xi+2}, I_{v C, 6 \xi+6}^{(o s)}=I_{v C, 6 \xi-6}, I_{v C, 6 \xi-6}^{(o s)}=I_{v C, 6 \xi+6}, I_{v C, 6 \xi}^{(o s)}=I_{v C, 6 \xi} \cdot I_{v C, k}^{(o s)}$ is the harmonic coefficient of current $i_{d c}$ forced in a system with the opposite sequence of the carrier.

Table 13 contains results of PAPF simulation tests without load for $f_{C}=10 \mathrm{kHz}$ with inductance $L_{-1}$ and $L_{-2}$ of equal or different value and symmetrical or asymmetrical supply voltages. The research shows that if the values of $L_{-1}$ and $L_{-2}$ and the values of the phase voltages of the grid are held to within $\pm 5 \%$, the rms value of current $i_{d c C}$ does not exceed $106 \%$ of the value calculated for the system with inductances $L_{-} 1$ and $L_{-} 2$ of equal value and symmetrical three-phase supply voltage.

Table 13. The rms value of currents of LCL-filter and dc-link capacitors for asymmetrical LCL-filter or the grid in an PAPF without load.

\begin{tabular}{|c|c|c|c|c|c|c|c|c|}
\hline \multirow{4}{*}{$M$} & \multicolumn{4}{|c|}{$U_{g 1(r m s)}^{\prime}=U_{g 2(r m s)}^{\prime}=U_{g 3(r m s)}^{\prime}$} & \multirow{2}{*}{\multicolumn{4}{|c|}{$\begin{array}{c}\left.U_{g 1(r m s)}^{\prime}=U_{g 2(r m s)}^{\prime}, U_{g 3(r m s)}^{\prime}=0.9 U_{g 1(r m s)}^{\prime}\right) \\
L_{-1}=76 \mu \mathrm{H} L_{-2}=84 \mu \mathrm{H}\end{array}$}} \\
\hline & \multicolumn{2}{|c|}{$L_{-1}=80 \mu \mathrm{H}, L_{-2}=80 \mu \mathrm{H}$} & \multicolumn{2}{|c|}{$L_{-1}=76 \mu \mathrm{H}, L_{-2}=84 \mu \mathrm{H}$} & & & & \\
\hline & $I_{c C 1, r m s}$ & $I_{d c C, r m s}$ & $I_{c C 1, r m s}$ & $I_{d c C, r m s}$ & $I_{c C 1, r m s}$ & $I_{c C 2, r m s}$ & $I_{c C 3, r m s}$ & $I_{d c C, r m s}$ \\
\hline & \multicolumn{3}{|c|}{ Results of Calculations/Results of Simulations Studies } & \multicolumn{5}{|c|}{ Results of Simulations Studies } \\
\hline 0.9 & $22.2 / 25.15$ & $53.0 / 53.5$ & $22.88 / 26.02$ & 53.77 & 24.18 & 26.42 & 26.51 & 56.77 \\
\hline 0.45 & $24.94 / 27.58$ & $53.0 / 55.48$ & $26.41 / 29.13$ & 55.63 & 29.77 & 28.99 & 28.99 & 56.24 \\
\hline 0.05 & $3.55 / 4.42$ & $56.5 / 56.58$ & $10.47 / 11.30$ & 56.75 & 11.41 & 11.27 & 11.26 & 56.64 \\
\hline
\end{tabular}

Simulation tests, the results of which are presented in Tables 11-13, were performed for sinusoidal (not containing higher harmonic) voltages $u_{g 1}^{\prime}, u_{g 2}^{\prime}$ and $u_{g 3}^{\prime}$.

\section{Experimental Results}

In order to verify the correctness of the analysis of components of the currents of the LCL-ripple filter capacitor and inductors, studies were conducted with the FAS-400k-400 and FA3-100k-400 active filters produced by the MEDCOM company based in Warsaw Jutrzenki 78A str., Poland. Tests with FAS-400k-400 were conducted in industrial conditions, in a coal mine where the PAPF works as a compensator of high harmonic currents consumed by a lifting machine in the mine with preset compensation of the 11th, 13th and 23rd harmonics. Filter parameters $U_{d c}, C_{d c}, C, L, f_{C}$, and $t_{d e a d}$ correspond with those assumed for the simulation research. Parameters of $\operatorname{Tr} 1$ are: transformer turns ratio $v_{z}$ is 26; rated low voltage is $400 \mathrm{~V}$, rated high voltage is $6 \mathrm{kV}$, rated power is $1.6 \mathrm{MVA}$, and short-circuit voltage is $5.68 \%$.

Figure 10a-c shows the research results of the PAPF for $f_{C}=15 \mathrm{kHz}$, while Figure $11 \mathrm{a}-\mathrm{c}$ shows the results for $f_{C}=10 \mathrm{kHz}$. The rms value of the LCL capacitor current shown in Figure 10c is 17.9 A, while this current shown in Figure 11a is 28 A. Figure 10a shows the oscilloscope waveform of output current $i_{01 \_1}(\mathrm{CH} 2)$. Figure $9 \mathrm{~b}$ shows the waveform of current $i_{01-1}$ obtained in the numerical model. The similarity of these waveforms $\left(\Delta i_{01 \_1, \max } \approx 100 \mathrm{~A}\right)$ proves the high precision of the numerical model and thus also the parameters listed in Tables 8-13. The rms value of the LCL capacitor current (16.2 A) determined from the equations derived in the article, taking into account the $5 \%$ tolerance of inductance of converter-side inductors, is $10 \%$ less than in the real system $(17.9 \mathrm{~A})$. 


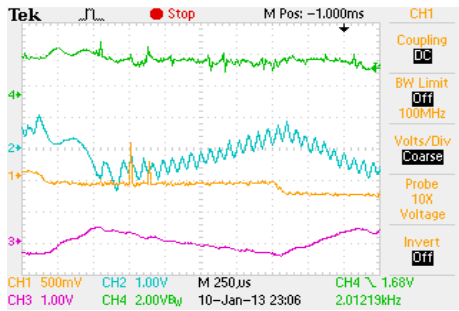

(a)

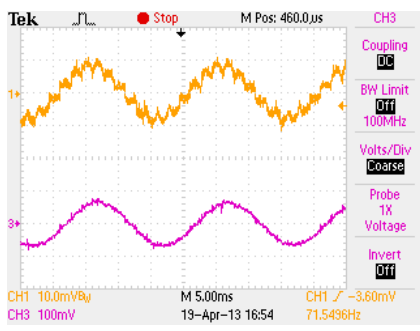

(b)

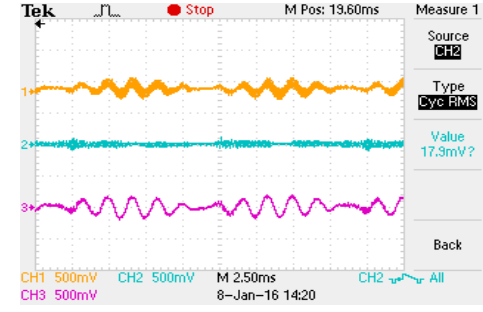

(c)

Figure 10. (a) Waveforms of load current $i_{L 1}(\mathrm{CH} 1: 45 \mathrm{~A} / \mathrm{div})$, output current $i_{01 \_1}(\mathrm{CH} 2: 200 \mathrm{~A} / \mathrm{div})$, setpoint signal of current $i_{1}(\mathrm{CH} 3: 1 \mathrm{~V} / \mathrm{div})$ and setpoint signal of current component $i_{1}$ to regulate the dc-link voltage $u_{d c}(\mathrm{CH} 4: 2 \mathrm{~V} / \mathrm{div})$; (b) waveforms of load current $i_{L 1}(\mathrm{CH} 1: 90 \mathrm{~A} / \mathrm{div})$ and input mains current $i_{g 1}(\mathrm{CH} 2: 120 \mathrm{~A} / \mathrm{div}) ;(\mathbf{c})$ waveforms of output current $i_{01 \_1}(\mathrm{CH} 1: 500 \mathrm{~A} / \mathrm{div})$, capacitor current $i_{c 1}\left(\mathrm{CH} 2: 500 \mathrm{~A} /\right.$ div) and PAPF output current $i_{p 1}(\mathrm{CH} 3: 500 \mathrm{~A} / \mathrm{div})$.

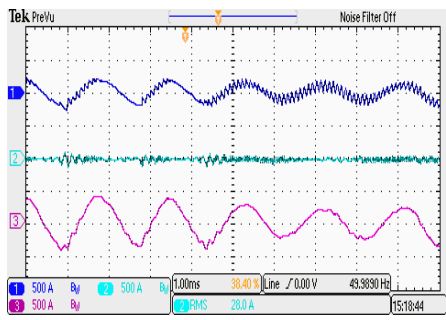

(a)

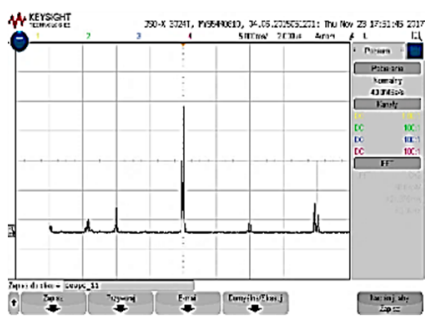

(b)

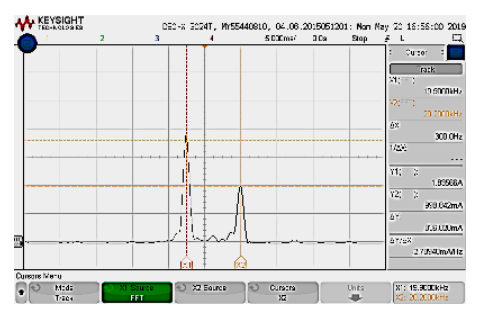

(c)

Figure 11. (a) Waveforms of output current $i_{01 \_1}(\mathrm{CH} 1: 500 \mathrm{~A} / \mathrm{div})$, capacitor current $i_{c 1}(\mathrm{CH} 2: 500 \mathrm{~A} / \mathrm{div})$ and PAPF output current $i_{p 1}(\mathrm{CH} 3: 500 \mathrm{~A} / \mathrm{div}) ;(\mathbf{b})$ harmonics spectrum of current $i_{d c}\left(8 \mathrm{~A}_{\mathrm{rms}} / \mathrm{div}\right)$ for the opposite sequence $u_{g 1,1}^{\prime}, u_{g 2,1}^{\prime}$, and $u_{g 3,1}^{\prime}$ voltages and wave carriers; (c) harmonics spectrum of current $i_{d c}\left(4 \mathrm{~A}_{\mathrm{rms}} / \mathrm{div}\right)$ for the same sequence $u_{g 1,1}^{\prime}, u_{g 2,1}^{\prime}$, and $u_{g 3,1}^{\prime}$ voltages and wave carriers (in an PAPF-type FA3-100k-400).

Figure $11 \mathrm{~b}$ shows the current $i_{d c}$ harmonic spectrum for a negative sequence carrier and $f_{C}=10 \mathrm{kHz}$ in an PAPF-type FAS-400k-400 with inductances $L_{-} 1=L_{-2}=L=80 \mu \mathrm{H}$, while Figure 11c shows current $i_{d c}$ harmonic spectrum for a positive sequence carrier and $f_{C}=10 \mathrm{kHz}$ in an PAPF-type FA3-100k-400 with inductances $L_{-1}=L_{-2}=L=200 \mu \mathrm{H}$.

On the basis of the oscillogram shown in Figure $11 \mathrm{~b}$, the current was obtained as $I_{v C, 2 \xi+2}^{(o s)}=35.2$ A. The value calculated by Equation (57) for the parameters of this filter $\left(U_{d c}=720 \mathrm{~V}, f_{C}=10 \mathrm{kHz}\right.$ and $L=80 \mu \mathrm{H}$ ) and the coefficients given in Table A1 for $M=0.9$ is $36.2 \mathrm{~A}$. Based on the oscillogram shown in Figure 11c, the following results were obtained for FA3-100k-400: $I_{v \mathrm{C}, 2 \xi-2(\mathrm{rms})}=14.7 \mathrm{~A}$; $I_{v C, 2 \xi+4(\mathrm{rms})}=7.98 \mathrm{~A}$. The calculated values (for $U_{d c}=720 \mathrm{~V}, f_{C}=10 \mathrm{kHz}$ and $L=200 \mu \mathrm{H}$ ) are $14.5 \mathrm{~A}$ and 7.82 A, respectively. The oscillograms shown in Figure 11b,c were made in the PAPF system without load. Figure 12 shows a photo of the current model of the FA3-400-400 filter.

The PAPF reduces the $\mathrm{THD}_{\mathrm{i}}$ factor of the consumed current of the $6 \mathrm{kV}$ grid from $14.7 \%$ to $4.8 \%$.

It is assumed that all elements of the examined energy system are normal or functional, which is not in line with the reality [33]. PAPF is an element of the system, the turn off of which from work results only in the deterioration of the quality of the voltage supplying the energy system. Switching the APF off in the system is safe because power of the device is 10 times lower than the power of the compensated load.

PAPF has systems for measuring the capacitance of dc-link circuit capacitors and LCL-filter capacitors when switching the device on and off, and continuous control of the rms value of LCL filter capacitor currents. The symmetry of current distribution of the respective branches of both inverters is also controlled. Exceeding any PAPF limit parameter results in switching off the device from the power supply system. 


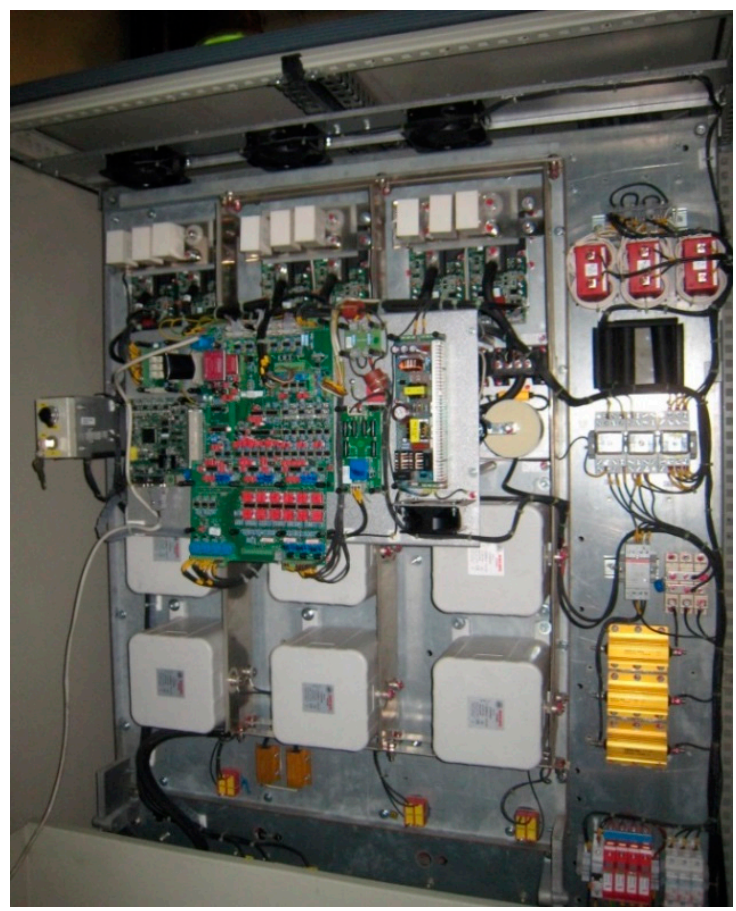

Figure 12. FA3-400k-400 installed in a coal mine.

\section{Conclusions}

Theoretical analysis and simulation research confirm a significant participation in PAPF systems with two parallel interleaved inverters of the LCL filter capacitor current component forced by non-linear load current and harmonics in the output current of a PAPF that compensates harmonics in the load. Simulation research supported by examples of calculations and experimental research confirm the validity of formulae describing different components of the capacitor current of the LCL-ripple filter, allowing for election of the capacitor from catalogues from the point of view of its effective current limit value. The analysis and relations presented allow calculation of the rms value of the LCL filter capacitor current.

If the PAPF does not compensate the reactive component of the fundamental harmonic, the share of harmonics current in the dc-link capacitor forced by the ripple currents in the inductors $L_{-} 1$ and $L_{-} 2$ increases significantly. In PAPF systems with two symmetrical inverters with output inductors $L$ and double three interleaved carriers, the dc-link capacitor current does not contain carrier harmonics of odd orders or their sideband harmonics.

Simulation studies show that if the values of inductance $L_{-1}$ and $L_{-2}$, and the phase voltages of the mains, are held within $\pm 5 \%$, the rms value of the current in the dc-link forced by the ripple current in the branches of the inverters does not exceed $106 \%$ of the value calculated for the symmetrical system.

The rms values of ripple currents in the LCL capacitors, LCL inductors and dc-link capacitors, which are sourced from the ripple current in the $L_{-} 1$ and $L_{-2}$ inductors, significantly depend on transistors' switching frequencies and on the value of the $L_{-} 1$ and $L_{-} 2$ inductances; however, they are not significantly dependent on the level of compensated power.

Supported by simulations, the analysis shows that if the sequence of three triangular carrier waves in the individual branches of the inverter is different from the harmonic sequence of the basic output voltages of the individual branches of the inverter, then the distribution of the spectrum of the harmonic currents in the dc-link capacitor forced by ripples of the output current of the inverter branches is changed; but this does not change the total effective value of this current.

The formulae describing the rms current of the LCL filter capacitor and the dc-link capacitor current given in the article may be applied to other topologies, such as a three-phase grid-connected 
converter (excluding the formula for the second mentioned component of the current of the capacitor of the LCL-filter), assuming that the $f_{C} / f_{g} \geq 40$ calculation error will not change.

On the basis of the simulation tests (only some results are given in the article), it can be concluded that for $f_{C} / f_{g} \geq 40$ :

- the rms values of currents in LCL filter capacitors in PAPF systems with double interleaved PWM carriers and double three interleaved PWM are similar, with accuracy of $1.5 \%$; and

- the rms values of total currents in LCL filter capacitors and dc-link capacitors in PAPF systems with a current control system with asymmetrical and symmetrical regular-sampled PWM are similar, with accuracy of $6 \%$.

It seems advisable to carry out a similar analysis for a PAPF containing one voltage inverter.

Funding: The author gratefully acknowledges the financial support provided by Institute of Control and Industrial Electronics (WUT).

Conflicts of Interest: The author declares no conflict of interest.

\section{Nomenclature}

$F_{m n}, F_{k} I_{m n}, I_{k}, U_{o 1, k}, U_{o 2, k}$

$\hat{F}_{m n}, \hat{F}_{k}, \hat{I}_{m n}, \hat{I}_{k}, \hat{U}_{o 1, k}, \hat{U}_{o 2, k}$,

$\hat{U}_{g, k^{\prime}}^{\prime} \hat{I}_{L, k}^{\prime}$

$I_{m n, r m s}, I_{m n(r m s)}, I_{k, r m s}, I_{k(r m s)}$

$Z_{c, k}, Z_{c, k}^{(0)}$

$i_{v, k_{s}+k}^{(\mathrm{comp})}$

$\varphi_{o, 2 \xi}, \varphi_{c, 2 \xi}^{(0)}$

$N$

$\lambda$

$v_{z}$
Fourier harmonic coefficients

Amplitudes of harmonic coefficients

Amplitudes of voltage and current harmonics brought to terminals 1, 2, 3 of

Tr1 transformer primary side

rms value of current harmonics

The circuit impedances for harmonics of positive and negative sequence and of zero sequence, respectively

The harmonic current component of the $\mathrm{k}_{\mathrm{s}} \pm \mathrm{k}$ order

The phase angles for harmonics of positive and negative sequence and of zero sequence, respectively

The number of operations in the microprocessor interruption service routine

Harmonic attenuation factor

Transformer turns ratio 
Appendix A

Table A1. The values of $H_{l}(M)$ and $G(M)$ coefficients.

\begin{tabular}{|c|c|c|c|c|c|c|c|c|c|c|c|c|c|c|c|}
\hline$M$ & $-H_{2 \xi-2}$ & $H_{2 \xi+4}$ & $-H_{4 \xi-4}$ & $-H_{4 \xi+2}$ & $-H_{6 \xi \pm 6}$ & $H_{6 \xi}$ & $-H_{8 \xi-8}$ & $H_{8 \xi-2}$ & $H_{8 \xi+4}$ & $H_{8 \xi}+10$ & $H_{10 \xi+2}$ & $H_{12 \xi}$ & $H_{14 \xi+4}$ & $H_{18 \xi}$ & $G(M)$ \\
\hline 0.00 & 0.000 & 0.000 & 0.000 & 0.000 & 0.000 & 0.486 & 0.000 & 0.000 & 0.000 & 0.000 & 0.000 & -0.246 & 0.000 & 0.158 & 0.567 \\
\hline 0.05 & 0.005 & 0.000 & 0.000 & 0.009 & 0.000 & 0.458 & 0.000 & 0.018 & -0.000 & 0.000 & -0.022 & -0.195 & 0.001 & 0.092 & 0.507 \\
\hline 0.10 & 0.019 & 0.000 & 0.000 & 0.036 & 0.000 & 0.381 & 0.000 & 0.063 & -0.002 & 0.000 & -0.073 & -0.074 & 0.010 & -0.023 & 0.403 \\
\hline 0.15 & 0.042 & 0.000 & 0.002 & 0.076 & 0.000 & 0.268 & 0.000 & 0.118 & -0.011 & 0.000 & -0.122 & 0.040 & 0.036 & -0.054 & 0.338 \\
\hline 0.20 & 0.073 & 0.001 & 0.005 & 0.126 & 0.001 & 0.140 & 0.000 & 0.158 & -0.028 & 0.000 & -0.137 & 0.085 & 0.068 & 0.002 & 0.312 \\
\hline 0.25 & 0.110 & 0.002 & 0.012 & 0.178 & 0.002 & 0.018 & 0.000 & 0.167 & -0.056 & 0.000 & -0.105 & 0.055 & 0.077 & 0.038 & 0.311 \\
\hline 0.30 & 0.151 & 0.005 & 0.023 & 0.227 & 0.005 & -0.079 & 0.001 & 0.139 & -0.088 & 0.000 & -0.039 & -0.008 & 0.047 & 0.009 & 0.335 \\
\hline 0.35 & 0.195 & 0.008 & 0.039 & 0.267 & 0.011 & -0.140 & 0.002 & 0.082 & -0.115 & 0.000 & 0.030 & -0.051 & -0.006 & -0.027 & 0.393 \\
\hline 0.40 & 0.238 & 0.014 & 0.060 & 0.294 & 0.020 & -0.159 & 0.005 & 0.014 & -0.128 & 0.001 & 0.072 & -0.047 & -0.042 & -0.014 & 0.446 \\
\hline 0.45 & 0.280 & 0.022 & 0.084 & 0.304 & 0.034 & -0.143 & 0.005 & -0.044 & -0.121 & 0.003 & 0.071 & -0.011 & -0.036 & 0.017 & 0.474 \\
\hline 0.50 & 0.317 & 0.033 & 0.112 & 0.297 & 0.051 & -0.102 & 0.017 & -0.076 & -0.092 & 0.006 & 0.035 & 0.025 & -0.002 & 0.016 & 0.485 \\
\hline 0.55 & 0.349 & 0.046 & 0.140 & 0.275 & 0.071 & -0.050 & 0.027 & -0.078 & -0.049 & 0.011 & -0.009 & 0.035 & 0.026 & -0.009 & 0.493 \\
\hline 0.60 & 0.374 & 0.063 & 0.166 & 0.240 & 0.091 & 0.000 & 0.038 & -0.055 & -0.001 & 0.019 & -0.038 & 0.019 & 0.025 & -0.015 & 0.504 \\
\hline 0.65 & 0.392 & 0.081 & 0.189 & 0.197 & 0.110 & 0.037 & 0.050 & -0.021 & 0.038 & 0.030 & -0.042 & -0.006 & 0.003 & 0.001 & 0.517 \\
\hline 0.70 & 0.401 & 0.102 & 0.206 & 0.150 & 0.123 & 0.057 & 0.050 & 0.011 & 0.060 & 0.043 & -0.025 & -0.019 & -0.016 & 0.012 & 0.529 \\
\hline 0.75 & 0.401 & 0.125 & 0.215 & 0.105 & 0.127 & 0.060 & 0.064 & 0.028 & 0.061 & 0.056 & -0.001 & -0.015 & -0.015 & 0.004 & 0.531 \\
\hline 0.80 & 0.394 & 0.148 & 0.217 & 0.065 & 0.121 & 0.050 & 0.063 & 0.030 & 0.046 & 0.066 & 0.017 & -0.003 & -0.002 & -0.006 & 0.521 \\
\hline 0.85 & 0.379 & 0.171 & 0.209 & 0.034 & 0.105 & 0.034 & 0.057 & 0.020 & 0.023 & 0.072 & 0.020 & 0.005 & 0.007 & -0.006 & 0.501 \\
\hline 0.90 & 0.358 & 0.193 & 0.194 & 0.013 & 0.083 & 0.019 & 0.046 & 0.006 & -0.001 & 0.072 & 0.013 & 0.005 & 0.006 & 0.001 & 0.475 \\
\hline 0.95 & 0.332 & 0.214 & 0.171 & 0.004 & 0.059 & 0.012 & 0.046 & -0.002 & -0.016 & 0.065 & 0.004 & 0.002 & 0.001 & 0.004 & 0.445 \\
\hline 1.00 & 0.301 & 0.232 & 0.143 & 0.007 & 0.038 & 0.014 & 0.018 & -0.001 & -0.019 & 0.053 & 0.001 & 0.000 & 0.001 & 0.002 & 0.414 \\
\hline
\end{tabular}




\section{References}

1. Liserre, M.; Blaabjerg, F.; Hansen, S. Design and Control of an LCL-Filter-Based Three-Phase Active Rectifier. IEEE Trans. Ind. Appl. 2005, 41, 1281-1291. [CrossRef]

2. Jeong, H.G.; Yoon, D.-K.; Lee, K.B. Design of an LCL-Filter for Three-Parallel Operation of Power Converters in Wind Turbines. J. Power Electron. 2013, 13, 437-445. [CrossRef]

3. Asiminoaei, L.; Aeloiza, E.; Enjeti, P.; Blaabjerg, F. Shunt Active-Power_Filter Topology Based on Parallel Interleaved Inverters. IEEE Trans. Ind. Electron. 2008, 55, 1175-1189. [CrossRef]

4. Jalili, K.; Bernet, S. Design of LCL Filters of Active-Front-End Two-Level Voltage-Source Converters. IEEE Trans. Ind. Electron. 2009, 56, 1674-1689. [CrossRef]

5. Sahoo, A.K.; Shahani, A.; Basu, K.; Mohan, N. LCL filter design for grid-connected inverters by analytical estimation of PWM ripple voltage. In Proceedings of the 2014 Twenty-Ninth Annual Applied Power Electronics Conference and Exposition (APEC), Fort Worth, TX, USA, 16-20 March 2014; pp. 1281-1286.

6. Tang, Y.; Loh, P.C.; Wang, P.; Choo, F.H.; Gao, F.; Blaabjerg, F. Generalized design of high performance shunt active power filter with output LCL filter. IEEE Trans. Ind. Electron. 2012, 59, 1443-1452. [CrossRef]

7. Vodyakho, O.; Mi, C.C. Three-level inverter-based shunt active power filter in three-phase three-wire and four-wire system. IEEE Trans. Power Electron. 2009, 24, 1350-1363. [CrossRef]

8. McGrath, B.P.; Holmes, D.G. A general analytical method for calculating inverter DC-link current harmonics. IEEE Trans. Ind. Appl. 2009, 45, 1851-1859. [CrossRef]

9. Kolar, J.W.; Wolbank, T.M.; Schrödl, M. Analytical calculation of the RMS current stress on the DC link capacitor of voltage DC link PWM converter systems. IEE Proc. Electr. Power Appl. 2006, 153, 535-543. [CrossRef]

10. Zhang, H.; Wheeler, N.; Grant, D. Switching harmonics in the DC link current in a PWM AC-DC-AC converter. In Proceedings of the IAS '95. Conference Record of the 1995 IEEE Industry Applications Conference Thirtieth IAS Annual Meeting, Orlando, FL, USA, 8-12 October 1995; pp. 2649-2655.

11. Zhang, D.; Wang, F.; Burgos, R.; Lai, R.; Boroyevich, D. DC-link ripple current reduction for paralleled three-phase voltage-source converters with interleaving. IEEE Trans. Power Electron. 2011, 26, 1741-1753. [CrossRef]

12. Ye, H.; Emadi, A. An interleaving scheme to reduce DC-link current harmonics of dual traction inverters in hybrid electric vehicles. In Proceedings of the 2014 IEEE Applied Power Electronics Conference and Exposition-APEC 2014, Fort Worth, TX, USA, 16-20 March 2014; pp. 3205-3211.

13. Quan, Z.; Li, Y. Impact of Carrier Phase Shift PWM on the DC Link Current of Single and Interleaved Three-Phase Voltage Source Converters. In Proceedings of the 2017 IEEE Energy Conversion Congress and Exposition (ECCE), Cincinnati, OH, USA, 1-5 October 2017; pp. 3851-3855.

14. Smolenski, R.; Jasinski, M.; Jarnut, M.; Bojarski, J.; Cecati, C. Compensation of CM voltage in systems consisting of interleaved AC-DC converters. In Proceedings of the 2014 IEEE 23rd International Symposium on Industrial Electronics (ISIE), Istanbul, Turkey, 1-4 June 2014; pp. 1996-2001.

15. Malinowski, M.; Bernet, S. A Simple Voltage Sensorless Active Damping Scheme for Three Phase PWM Converters with an LCL Filter. IEEE Trans. Ind. Electron. 2008, 55, 1876-1880. [CrossRef]

16. Wu, D.; Chen, Y.; Hong, S.; Zhao, X.; Luo, J.; Gu, Z. Mathematical model analysis and LCL Filter Design of VSC. In Proceedings of the 7th International Power Electronics and Motion Control Conference, Harbin, China, 2-5 June 2012; pp. 2799-2804.

17. Lang, Y.; Xu, D.; Hadianamrei, S.R.; Ma, H. A novel design method of LCL type utility interface for three-phase voltage source rectifier. In Proceedings of the 2005 IEEE 36th Power Electronics Specialists Conference, Recife, Brazil, 16 June 2005; pp. 313-317.

18. Shen, G.; Zhu, X.; Zhang, J.; Xu, D. A New Feedback Method for PR Current Control of LCL-Filter-Based Grid-Connected Inverter. IEEE Trans. Ind. Electron. 2010, 57, 2033-2041. [CrossRef]

19. Pei, X.; Zhou, W.; Kang, Y. Analysis and calculation of DC-link current and voltage ripples for three-phase inverter with unbalanced load. IEEE Trans. Power Electron. 2015, 30, 5401-5412. [CrossRef]

20. Lascu, C.; Asiminoaei, L.; Boldea, L.; Blaabjerg, F. Frequency response analysis of current controllers for selective harmonic compensation in active power filters. IEEE Trans. Ind. Electron. 2009, 56, 337-347. [CrossRef]

21. Ben-Sheng, C.; Yuan-Yih, H. A minimal harmonic controller for a STATCOM. IEEE Trans. Ind. Electron. 2008, 55, 655-664. 
22. Platek, T.; Osypinski, T. Current Control with Asymmetrical Regular Sampled PWM Modulator Applied for Parallel Active Filter. Bull. Pol. Acad. Sci. Tech. 2016, 64, 287-300.

23. Holmes, D.G.; Lipo, T.A. Pulse Width Modulation For Power Converters; IEEE PRESS: Hoboken NJ 07030 USA, Willey-Interscience; 2003.

24. Zhang, D.; Wang, F.; Burgos, R.; Lai, R.; Boroyevich, D. Impact of interleaving on ac passive components of paralleled three-phase voltage-source converters. IEEE Trans. Ind. Appl. 2010, 46, 1042-1054. [CrossRef]

25. Rockhill, A.A.; Liserre, M.; Teodorescu, R.; Rodriguez, P. Grid-Filter Design for a Multimegawatt Medium-Voltage Voltage-Source Inverter. IEEE Trans. Ind. Electron. 2011, 58, 1205-1217. [CrossRef]

26. Shang, Y. Synchronization in networks of coupled harmonic oscillators with stochastic perturbation and time delays. Ann. Acad. Rom. Sci. Ser. Math. Appl. 2012, 4, 44-58.

27. Holmes, D.G.; Lipo, T.A.; McGrath, B.P.; Kong, W.Y. Optimized design of stationary frame three phase AC current regulators. IEEE Trans. Power Electron. 2009, 24, 2417-2426. [CrossRef]

28. Liu, F.; Zha, X.; Zhou, Y.; Duan, S. Design and research on parameter of LCL filter in three-phase grid-connected inverter. In Proceedings of the 2009 IEEE 6th International Power Electronics and Motion Control Conference, Wuhan, China, 17-20 May 2009; pp. 2174-2177.

29. Liu, Y.; Lai, C.M. LCL Filter Design with EMI Noise Consideration for Grid-Connected Inverter. Energies 2018, 11, 1646. [CrossRef]

30. Abusara, M.A.; Jamil, M.; Sharkh, S.M. Repetitive current control of an interleaved grid connected inverter. In Proceedings of the 20123 rd IEEE International Symposium on Power Electronics for Distributed Generation Systems (PEDG), Aalborg, Denmark, 25-28 June 2012; pp. 558-563.

31. Jiang, Y.; Ekström, A. General Analysis of Harmonic Transfer Through Converters. IEEE Trans. Power Electron. 1997, 12, 287-293. [CrossRef]

32. Kimball, J.W.; Zawodniok, M. Reducing Common-Mode Voltage in Three-Phase Sine-Triangle PWM with Interleaved Carriers. In Proceedings of the 2010 Twenty-Fifth Annual IEEE Applied Power Electronics Conference and Exposition (APEC), Palm Springs, CA, USA, 21-25 February 2010; Volume 26, pp. 2229-2236.

33. Shang, Y. Resilient Multiscale Coordination Control against Adversarial Nodes. Energies 2018, 11, 1844. [CrossRef]

(C) 2019 by the author. Licensee MDPI, Basel, Switzerland. This article is an open access article distributed under the terms and conditions of the Creative Commons Attribution (CC BY) license (http://creativecommons.org/licenses/by/4.0/). 